\title{
A characterization of infinite smooth Lyndon words
}

\author{
Geneviève Paquin ${ }^{\dagger}$ \\ Laboratoire de mathématiques, Université de Savoie, CNRS UMR 5127, 73376 Le Bourget du Lac, France. E-mail: \\ Genevieve.Paquin@univ-savoie.fr
}

received $25^{\text {th }}$ September 2008 , revised $19^{\text {th }}$ September 2010, accepted $22^{\text {nd }}$ October 2010.

In a recent paper, Brlek, Jamet and Paquin showed that some extremal infinite smooth words are also infinite Lyndon words. This result raises a natural question: are they the only ones? If no, what do the infinite smooth words that are also Lyndon words look like? In this paper, we give the answer, proving that the only infinite smooth Lyndon words are $m_{\{a<b\}}$, with $a, b$ even, $m_{\{1<b\}}$ and $\Delta_{1}^{-1}\left(m_{\{1<b\}}\right)$, with $b$ odd, where $m_{\mathcal{A}}$ is the minimal infinite smooth word with respect to the lexicographic order over a numerical alphabet $\mathcal{A}$ and $\Delta$ is the run-length encoding function.

Keywords: Lyndon words, Lyndon factorization, smooth words, extremal smooth words

\section{Introduction}

A finite Lyndon word is easy to define: it is a finite word that is smaller than any of its suffixes. The class of finite Lyndon words was first introduced by Chen, Fox and Lyndon in [CFL58] for the construction of bases of the lower central series for free groups. The authors proved that any finite word can be expressed as a unique non-increasing product (concatenation) of Lyndon words, which they called the Lyndon factorization. Lyndon words were then studied by Duval [Duv83, Duv88]. Among his results is an algorithm that generates Lyndon words of bounded length for a finite alphabet and another one that computes the Lyndon factorization in linear time with respect to the length of the word. Siromoney et al. [SMDS94] later defined infinite Lyndon words as infinite words smaller than any of their suffixes, in order to introduce Lyndon factorization of infinite words. Lyndon words also appeared for instance in [Lot83, MR89, Reu93]. The Lyndon factorization gives nice properties about the structure of words. Since a few years, a wide literature is devoted to Lyndon words: [BdL97, HR03, Reu07, Ric07, Séé03]. In particular, Melançon [Mel00] studied Lyndon factorization of Sturmian infinite words and gave a formula that completely describes the Lyndon factorization of any characteristic Sturmian word.

The smooth infinite words over $\mathcal{A}=\{1,2\}$ form an infinite class $\mathcal{K}$ of infinite words containing the well-known Kolakoski word $K$ [Kol65] defined as one of the two fixed points of the run-length encoding function $\Delta$, that is

$$
\Delta(K)=K=2211212212211211221211212211211212212211212212 \cdots .
$$

\footnotetext{
${ }^{\dagger}$ Supported by the Fonds de recherche sur la nature et les technologies (Québec, Canada) 
They are characterized by the property that the orbit obtained by iterating $\Delta$ is contained in $\{1,2\}^{\omega}$. In the early work of Dekking [Dek81], there are some challenging conjectures on the structure of $K$ that still remain unsolved despite the efforts devoted to the study of patterns in $K$. For instance, we know from Carpi [Car94] that $K$ and more generally, any word in the infinite class $\mathcal{K}$ of smooth words over $\mathcal{A}=\{1,2\}$, contain only a finite number of squares, implying by direct inspection that $K$ and any $w \in \mathcal{K}$ are cube-free. Weakley [Wea89] showed that the number of factors of length $n$ of $\mathcal{K}$ is polynomially bounded. In [BL03], a connection was established between the palindromic complexity and the recurrence of $K$. Then Berthé, Brlek and Choquette [BBC05] studied smooth words over arbitrary alphabets and obtained a new characterization of the infinite Fibonacci word $F$. Relevant work may also be found in [BBBLP03] and in [BBC05, JP05], where generalized Kolakoski words are studied for arbitrary alphabets. The authors investigated in [BMP07] the extremal infinite smooth words, that is the minimal and the maximal ones w.r.t. the lexicographic order, over the alphabets $\{1,2\}$ and $\{1,3\}$ : a surprising link is established between $F$ and the minimal infinite smooth word over $\{1,3\}$.

More recently, Brlek, Jamet and Paquin [BJP08] studied the extremal smooth words for any two-letter numerical alphabet and they showed the existence of infinite smooth words that are also Lyndon words: the minimal smooth word over an even alphabet and the one over the alphabet $\{1, b\}$, with $b$ odd, are Lyndon words. Then a natural question arises: are there other infinite smooth words that are also infinite Lyndon words?

In this paper, we show that the only infinite smooth words that are also Lyndon words are related to the minimal smooth Lyndon words given in [BJP08]. In order to prove it, we study the words over a two-letter alphabet depending on the parity of the letters. The paper is organized as follows. In Section 2, we recall the basic definitions in combinatorics on words, we state the notation we will use next and we give useful known results. Section 3 is devoted to the characterization of infinite smooth Lyndon words. It is divided in four subsections. In Section 3.1, we study the case of an alphabet $\mathcal{A}=\{a<b\}$, with $a$ even and $b$ odd. We show that there is no infinite Lyndon words that is also smooth. In Section 3.2, we are interested in even alphabets. We show that only the minimal smooth word is a Lyndon word. Section 3.3 is devoted to odd alphabets. We prove that only $m_{\{1, b\}}$ and $\Delta_{1}^{-1}\left(m_{\{1, b\}}\right)$ are Lyndon words. Finally, Section 3.4 studies the words over an alphabet $\{a<b\}$, with $a$ odd and $b$ even. In this last case, we show that there is no infinite Lyndon word that is also smooth.

This paper is an extended version of a paper presented in Prague (Czech Republic) during the 13th Prague Stringology Conference [Paq08a]. In this new version, we give the complete proofs. We also correct our main result, since Proposition 24 in [Paq08a] appeared to be false. That leads to the third form of smooth Lyndon words, namely $\Delta_{1}^{-1}\left(m_{\{1, b\}}\right)$, with $b$ odd.

\section{Preliminaries}

Throughout this paper, $\mathcal{A}$ is a finite alphabet of letters equipped with a total order $<$. A finite word $w$ is a finite sequence of letters $w=w[0] w[1] \cdots w[n-1]$, where $w[i] \in \mathcal{A}$ denotes its $(i+1)$-th letter. Its length is $n$ and we write $|w|=n$. The set of $n$-length words over $\mathcal{A}$ is denoted by $\mathcal{A}^{n}$. By convention the empty word is denoted by $\varepsilon$ and its length is 0 . The free monoid generated by $\mathcal{A}$ is defined by $\mathcal{A}^{*}=\bigcup_{n \geq 0} \mathcal{A}^{n}$ and $\mathcal{A}^{*} \backslash \varepsilon$ is denoted by $\mathcal{A}^{+}$. The set of right infinite words, also called infinite words for short, is denoted by $\mathcal{A}^{\omega}$ and $\mathcal{A}^{\infty}=\mathcal{A}^{*} \cup \mathcal{A}^{\omega}$. Adopting a consistent notation for finite words over the infinite alphabet $\mathbb{N}$, $\mathbb{N}^{*}=\bigcup_{n \geq 0} \mathbb{N}^{n}$ is the set of finite words and $\mathbb{N}^{\omega}$ is that of infinite ones. Given a word $w \in \mathcal{A}^{*}$, a factor $f$ 
of $w$ is a word $f \in \mathcal{A}^{*}$ satisfying

$$
\exists x, y \in \mathcal{A}^{*}, w=x f y .
$$

If $x=\varepsilon$ (resp. $y=\varepsilon$ ) then $f$ is called a prefix (resp. suffix). Note that by convention, the empty word is a suffix and a prefix of any word. A block of length $k$ is a maximal factor of the particular form $f=\alpha^{k}$, with $\alpha \in \mathcal{A}$. The set of all factors of $w$, also called the language of $w$, is denoted by $F(w)$ and those of length $n$ is $F_{n}(w)=F(w) \cap \mathcal{A}^{n}$. We denote by $\operatorname{Pref}(w)$ (resp. $\operatorname{Suff}(w)$ ) the set of all prefixes (resp. suffixes) of $w$.

Over an arbitrary two-letter alphabet $\mathcal{A}=\{a, b\}$, there is a usual length preserving morphism, the complementation, defined by $\bar{a}=b, \bar{b}=a$, which extends to words as follows. The complement of $u=u[0] u[1] \cdots u[n-1] \in \mathcal{A}^{n}$ is the word $\bar{u}=\overline{u[0]} \overline{u[1]} \cdots \overline{u[n-1]}$. The reversal of $u$ is the word $\widetilde{u}=u[n-1] \cdots u[1] u[0]$.

For $u, v \in \mathcal{A}^{*}$, we write $u<v$ if and only if $u$ is a proper prefix of $v$ or if there exists an integer $k$ such that $u[i]=v[i]$ for $0 \leq i \leq k-1$ and $u[k]<v[k]$. The relation $\leq$ defined by $u \leq v$ if and only if $u=v$ or $u<v$, is called the lexicographic order. That definition holds for $\mathcal{A}^{\omega}$. Note that in general the complementation does not preserve the lexicographic order. Indeed, when $u$ is not a proper prefix of $v$ then

$$
u>v \Longleftrightarrow \bar{u}<\bar{v} .
$$

\subsection{Lyndon words and factorizations}

A word $u \in \mathcal{A}^{*}$ is a Lyndon word if $u<v$ for all proper non-empty suffixes $v$ of $u$. For instance, the word 11212 is a Lyndon word while 12112 is not since $112<12112$. A word of length 1 is clearly a Lyndon word. The set of Lyndon words is denoted by $\mathcal{L}$.

From the works of Chen, Fox and Lyndon, we have the following theorem.

Theorem 1 ([CFL58]) Any non-empty finite word $w$ is uniquely expressed as a non-increasing product of Lyndon words

$$
w=\ell_{0} \ell_{1} \cdots \ell_{n}=\bigodot_{i=0}^{n} \ell_{i}, \text { with } \ell_{i} \in \mathcal{L} \text { and } \ell_{0} \geq \ell_{1} \geq \cdots \geq \ell_{n} .
$$

Siromoney et al. [SMDS94] extended Theorem 1 to infinite words. The set $\mathcal{L}_{\infty}$ of infinite Lyndon words consists of infinite words smaller than any of their suffixes.

Theorem 2 ([SMDS94]) Any infinite word $w$ is uniquely expressed as a non-increasing product of Lyndon words, finite or infinite, in one of the two following forms:

i) either there exists an infinite sequence $\left(\ell_{k}\right)_{k \geq 0}$ of elements in $\mathcal{L}$ such that

$$
w=\ell_{0} \ell_{1} \ell_{2} \cdots \text { and for all } k, \ell_{k} \geq \ell_{k+1}
$$

ii) there exist a finite sequence $\ell_{0}, \ldots, \ell_{m}(m \geq 0)$ of elements in $\mathcal{L}$ and $\ell_{m+1} \in \mathcal{L}_{\infty}$ such that

$$
w=\ell_{0} \ell_{1} \cdots \ell_{m} \ell_{m+1} \text { and } \ell_{0} \geq \cdots \geq \ell_{m}>\ell_{m+1} .
$$


Let us recall from ([Lot83] Chapter 5.1) a useful property concerning Lyndon words.

Lemma 3 Let $u, v \in \mathcal{L}$. Then $u v \in \mathcal{L}$ if and only if $u<v$.

A direct corollary of this lemma is:

Corollary 4 Let $u, v \in \mathcal{L}$, with $u<v$. Then $u v^{n}, u^{n} v \in \mathcal{L}$, for all $n \geq 0$.

\subsection{Run-length encoding}

The widely known run-length encoding is used in many applications as a method for compressing data. For instance, the first step in the algorithm used for compressing the data transmitted by Fax machines consists of a run-length encoding of each line of pixels. Let $\mathcal{A}=\{a<b\}$ be an ordered alphabet. Then every word $w \in \mathcal{A}^{*}$ can be uniquely written as a product of factors as follows:

$$
w=a^{i_{0}} b^{i_{1}} a^{i_{2}} \ldots \quad \text { or } \quad w=b^{i_{0}} a^{i_{1}} b^{i_{2}} \ldots
$$

with $i_{k} \geq 1$ for $k \geq 0$. The operator giving the size of the blocks appearing in the coding is a function $\Delta: \mathcal{A}^{*} \longrightarrow \mathbb{N}^{*}$, defined by $\Delta(w)=i_{0}, i_{1}, i_{2}, \cdots$ which is easily extended to infinite words as $\Delta$ : $\mathcal{A}^{\omega} \longrightarrow \mathbb{N}^{\omega}$.

For instance, let $\mathcal{A}=\{1,3\}$ and $w=13333133111$. Then

$$
w=1^{1} 3^{4} 1^{1} 3^{2} 1^{3} \quad \text { and } \quad \Delta(w)=[1,4,1,2,3] .
$$

When $\Delta(w) \subseteq\{1,2, \cdots, 9\}^{*}$, the punctuation and the parentheses are often omitted in order to manipulate the more compact notation $\Delta(w)=14123$. This example is a special case where the coding integers do not coincide with the alphabet on which is encoded $w$, so that $\Delta$ can be viewed as a partial function

$$
\Delta:\{1,3\}^{*} \longrightarrow\{1,2,3,4\}^{*} .
$$

From now on, we only consider two-letter alphabets $\mathcal{A}=\{a<b\}$, with $a, b \in \mathbb{N} \backslash\{0\}$.

Recall from [BL03] that $\Delta$ is not bijective since $\Delta(w)=\Delta(\bar{w})$, but commutes with the reversal $(\sim)$, is stable under complementation $\left(^{-}\right)$and preserves palindromicity. Since $\Delta$ is not bijective, pseudo-inverse functions

$$
\Delta_{a}^{-1}, \Delta_{b}^{-1}: \mathcal{A}^{*} \longrightarrow \mathcal{A}^{*}
$$

are defined for two-letter alphabets by

$$
\Delta_{\alpha}^{-1}(u)=\alpha^{u[0]} \bar{\alpha}^{u[1]} \alpha^{u[2]} \bar{\alpha}^{u[3]} \ldots, \quad \text { for } \alpha \in\{a, b\} .
$$

Note that the pseudo-inverse function $\Delta^{-1}$ also commutes with the mirror image, that is

$$
\widetilde{\Delta_{\alpha}^{-1}(w)}=\Delta_{\beta}^{-1}(\widetilde{w}),
$$

where $\beta=\alpha$ if $|w|$ is odd and $\beta=\bar{\alpha}$ if $|w|$ is even.

The operator $\Delta$ may be iterated, provided the process is stopped when the coding alphabet changes or when the resulting word has length 1 . 
Example 5 Let $w=1333111333133311133313133311133313331113331$. The successive application of $\Delta$ gives:

$$
\begin{aligned}
& \Delta^{0}(w)=1333111333133311133313133311133313331113331 \\
& \Delta^{1}(w)=1333133311133313331 \\
& \Delta^{2}(w)=131333131 \\
& \Delta^{3}(w)=1113111 \\
& \Delta^{4}(w)=\mathbf{3} 13 \\
& \Delta^{5}(w)=111 \\
& \Delta^{6}(w)=\mathbf{3} \text {. }
\end{aligned}
$$

\subsection{Smooth words}

The set of finite smooth words over the alphabet $\mathcal{A}=\{a<b\}$ is defined by

$$
\Delta_{\mathcal{A}}^{+}=\left\{w \in \mathcal{A}^{*} \mid \exists n \in \mathbb{N}, \Delta^{n}(w)=\alpha \in \mathcal{A} \text { and } \forall k \leq n, \Delta^{k}(w) \in \mathcal{A}^{*}\right\} .
$$

The operator $\Delta$ extends to infinite words (see [BL03]). Define the set of infinite smooth words over $\mathcal{A}=\{a<b\}$ by

$$
\mathcal{K}_{\mathcal{A}}=\left\{w \in \mathcal{A}^{\omega} \mid \forall k \in \mathbb{N}, \Delta^{k}(w) \in \mathcal{A}^{\omega}\right\} .
$$

The well-known [Kol65] Kolakoski word denoted by $K$ is defined as the fix-point starting with the letter 2 of the operator $\Delta$ over the alphabet $\{1,2\}$ :

$$
K=2211212212211211221211212211211212212211 \cdots .
$$

More generally, the operator $\Delta$ has two fix-points in $\mathcal{K}_{\mathcal{A}}$, namely

$$
\Delta\left(K_{(a, b)}\right)=K_{(a, b)} \quad \text { and } \quad \Delta\left(K_{(b, a)}\right)=K_{(b, a)},
$$

where $K_{(a, b)}$ is the generalized Kolakoski word [JP05] over the alphabet $\{a, b\}$ starting with the letter $a$.

Example 6 The Kolakoski word over $\mathcal{A}=\{1,2\}$ starting with the letter 2 is $K=K_{(2,1)}$. We also have $K_{(2,3)}=22332223332233223332 \cdots$ and $K_{(3,1)}=33311133313133311133 \cdots$.

A bijection $\Phi: \mathcal{K}_{\mathcal{A}} \longrightarrow \mathcal{A}^{\omega}$ is defined by

$$
\Phi(w)=\Delta^{0}(w)[0] \Delta^{1}(w)[0] \Delta^{2}(w)[0] \cdots=\prod_{i \geq 0} \Delta^{i}(w)[0]
$$

and its inverse is defined as follows. Let $u \in \mathcal{A}^{k}$, then $\Phi^{-1}(u)=w_{k}$, where

$$
w_{n}= \begin{cases}u[k-1], & \text { if } n=1 \\ \Delta_{u[k-n]}^{-1}\left(w_{n-1}\right), & \text { if } 2 \leq n \leq k .\end{cases}
$$

Then for $k=\infty, \Phi^{-1}(u)=\lim _{k \rightarrow \infty} w_{k}=\lim _{k \rightarrow \infty} \Phi^{-1}(u[0 \ldots k-1])$. 
Remark 7 With respect to the usual topology defined by

$$
d\left(\left(u_{n}\right)_{n \geq 0},\left(v_{n}\right)_{n \geq 0}\right):=2^{-\min \left\{j \in \mathbb{N}, u_{j} \neq v_{j}\right\}},
$$

the previous limit exists since $w_{k}$ is a prefix of $w_{k+1}$ for all $k \in \mathbb{N}$.

Example 8 For the word $w=1333111333133311133313133311133313331113331$ of the Example 5, $\Phi(w)=1111313$.

Note that since $\Phi$ is a bijection between the infinite smooth words and the infinite words, the set of infinite smooth words is infinite. Moreover, given a prefix of $\Phi(w)$ with $w$ a smooth word, we can construct a prefix of $w$ as in the following example.

Example 9 Let $p=1221$ be a prefix of $\Phi(w)$, with $w \in\{1,2\}^{\omega}$ an infinite smooth word. Then we compute from bottom to top, using the $\Delta^{-1}$ operator:

$$
\begin{aligned}
\Delta^{0}(w) & =11221221 \cdots \\
\Delta^{1}(w) & =2212 \cdots \\
\Delta^{2}(w) & =21 \cdots \\
\Delta^{3}(w) & =1 \cdots
\end{aligned}
$$

Note that in $\Delta^{2}(w)$, the letter 1 is obtained by deduction, since $\Delta^{3}(w)$ indicates that the first block of letters of $\Delta^{2}(w)$ has length 1 . The last written letter of every line is deduced by a similar argument.

We recall from [BMP07] the useful right derivative $D_{r}: \mathcal{A}^{*} \rightarrow \mathbb{N}^{*}$ defined by

$$
D_{r}(w)= \begin{cases}\varepsilon & \text { if } \Delta(w)=\alpha, \alpha<b \text { or } w=\varepsilon, \\ \Delta(w) & \text { if } \Delta(w)=x b, \\ x & \text { if } \Delta(w)=x \alpha, \alpha<b,\end{cases}
$$

where $\alpha \in \mathbb{N}$ and $x \in \mathcal{A}^{*}$. A word $w$ is $r$-smooth (also called a smooth prefix) if $\forall k \geq 0, D_{r}^{k}(w) \in \mathcal{A}^{*}$. In other words, if a word $w$ is $r$-smooth, then it is a prefix of at least one infinite smooth word (see [Paq08b] for more details).

Example 10 Let $w=112112212$. Then $\Delta(w)=212211, \Delta^{2}(w)=1122, \Delta^{3}(w)=22, \Delta^{4}(w)=2$ and $D_{r}(w)=21221, D_{r}^{2}(w)=112, D_{r}^{3}(w)=2$.

Similarly, the operator $D$ is defined over the alphabet $\{a<b\}$ by

$$
D(w)= \begin{cases}\varepsilon & \text { if } \Delta(w)<b \text { or } w=\varepsilon, \\ \Delta(w) & \text { if } \Delta(w)=b x b \text { or } \Delta(w)=b, \\ b x & \text { if } \Delta(w)=b x u, \\ x b & \text { if } \Delta(w)=u x b, \\ x & \text { if } \Delta(w)=u x v,\end{cases}
$$

where $u$ and $v$ are blocks of length $<b$. A finite word is called a smooth factor (also called a $C^{\infty}$-word in [Car94, Hua97, Hua08, Wea89]) if there exists $k \in \mathbb{N}$ such that $D^{k}(w)=\varepsilon$ and $\forall j<k, D^{j}(w) \in \mathcal{A}^{*}$. 


\subsection{Known results}

The minimal (resp. the maximal) infinite smooth word over the alphabet $\mathcal{A}$ is the smallest (resp. largest) infinite smooth word, with respect to the lexicographic order. It is denoted by $m_{\mathcal{A}}$ (resp. $M_{\mathcal{A}}$ ).

An alphabet $\mathcal{A}=\{a<b\}$ is called an odd alphabet (resp. even alphabet) if both $a$ and $b$ are odd (resp. even). The extremal smooth words satisfy the following properties established in a previous paper.

Proposition 11 ([BJP08]) Let $\mathcal{A}=\{a<b\}$. Then the following properties hold.

i) If $a$ and $b$ are both even we have $\Phi\left(M_{\{a, b\}}\right)=b^{\omega}, \Phi\left(m_{\{a, b\}}\right)=a b^{\omega}$ and $m_{\{a, b\}} \in \mathcal{L}_{\infty}$.

ii) If $a$ and $b$ are both odd we have $\Phi\left(M_{\{a, b\}}\right)=(b a)^{\omega}, \Phi\left(m_{\{a, b\}}\right)=(a b)^{\omega}$ and $m_{\{a, b\}} \in \mathcal{L}_{\infty} \Longleftrightarrow$ $a=1$.

Let us recall useful results about smooth words.

Lemma 12 ([BBC05]) Let $u, v$ be finite smooth words. If there exists an index $m$ such that, for all $i$, $0 \leq i \leq m$, the last letter of $\Delta^{i}(u)$ differs from the first letter of $\Delta^{i}(v)$, and $\Delta^{i}(u) \neq 1, \Delta^{i}(v) \neq 1$, then

$$
\begin{aligned}
\Phi(u v) & =\Phi(u)[0 \ldots m] \cdot \Phi \circ \Delta^{m+1}(u v) \\
\Delta^{i}(u v) & =\Delta^{i}(u) \Delta^{i}(v) .
\end{aligned}
$$

The following properties follow immediately from the definitions. For more details, the reader is referred to [Paq08b].

Recall from [BDLV06] that in the case of the alphabet $\mathcal{A}=\{1,2\}$, every finite smooth word $w \in \Delta_{\mathcal{A}}^{+}$ can be easily extended to the right in a smooth word by means of the function $\Phi$ as $\forall u \in \mathcal{A}^{\infty}, w \in$ $\operatorname{Pref}\left(\Phi^{-1}(\Phi(w) \cdot u)\right)$. Its generalization to arbitrary alphabets is immediate (see [Paq08b]).

Proposition 13 ([Paq08b]) Let $\mathcal{A}=\{a<b\}$. Then the following properties hold.

i) Any smooth prefix can be arbitrarily right extended to an infinite smooth word.

ii) Let $u=\Phi(w)$, with $w \in \mathcal{A}^{\omega}$ an infinite smooth word. If $u=u^{\prime} u^{\prime \prime}$, then $\Phi^{-1}\left(u^{\prime}\right)$ is a prefix of $w$.

\section{Characterization of infinite smooth Lyndon words}

In this section, we prove our main result: the only infinite smooth words that are also infinite Lyndon words are $m_{\{a<b\}}$, with $a, b$ even, $m_{\{1<b\}}$ and $\Delta_{1}^{-1}\left(m_{\{1<b\}}\right)$, with $b$ odd.

In order to prove it, we study the four possible combinations of the parity of the letters $a$ and $b$. For each case, we fix a length $n$ and we then consider all the possible words $p$ of length $\leq n$ such that $\Phi^{-1}(p)$ is a prefix of an infinite smooth word $w$. We suppose that $w$ is also a Lyndon word. Then for each word $p$, either we prove that $\Phi^{-1}(p)$ cannot be a prefix of a Lyndon word by showing the existence of a smaller suffix, or we describe an infinite smooth Lyndon word having $\Phi^{-1}(p)$ as a prefix. For each case, the different values of $p$ are illustrated by a path in a tree.

Lemma 14 Let $p \in\{a<b\}^{*}$ be such that $\Phi^{-1}(p)$ is a prefix of an infinite smooth Lyndon word $w$. Then $p[0]=a$. 
Proof: Follows from the equality $p[0]=w[0]$ and since a Lyndon word $w$ must start by the smallest letter, namely $a$.

Lemma 14 will be used in this section in order to exclude the cases numbered (0) in the proofs.

\subsection{Over $\mathcal{A}$ with $a$ even and $b$ odd}

In this section, we prove the following result.

Theorem 15 Over the alphabet $\{a<b\}$, with a even and $b$ odd, there is no infinite smooth word that is also a Lyndon word.

Proof: Figure 1 illustrates the 6 possible cases to consider, using a tree. The leaves correspond to the first letter of $\Phi(w)$ that leads to a contradiction: the prefix $\Phi^{-1}(p)$ obtained cannot be the prefix of an infinite Lyndon word. We will prove it by showing that there exists a factor $f$ of $w$ not prefix of $\Phi^{-1}(p)$ such that $f<w$. For clarity issues, the first letter of $f$ is underlined in $w$.

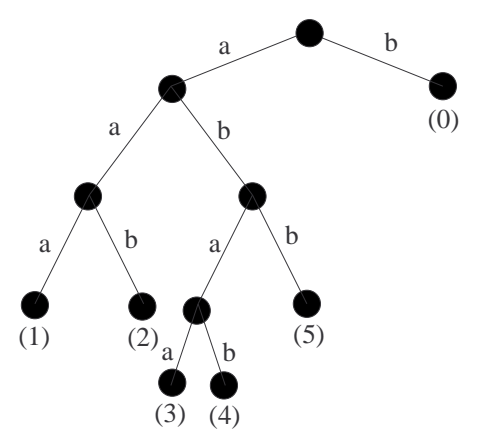

Figure 1: Possible cases for an even-odd alphabet

Case (1) If $p=a a a$, then

$$
\begin{aligned}
\Delta^{0}(w) & =\left(a^{a} b^{a}\right)^{\frac{a}{2}}\left(\underline{a}^{b} b^{b}\right)^{\frac{a}{2}} \ldots \\
\Delta^{1}(w) & =a^{a} b^{a} \cdots \\
\Delta^{2}(w) & =a a \cdots
\end{aligned}
$$

Since $w$ has the prefix $a^{a} b^{a}$ and the factor $f=a^{b}$, it cannot be a Lyndon word.

Remark 16 Since the smallest letter $a$ of the alphabet is even, $2 \leq a \leq p[3]$. That allows us to assume that $\Delta^{2}(w)$ starts with a block of length at least 2. This argument holds for $\Delta^{i}(w)$, $i \geq 0$, and will be used for almost all the cases considered in this paper.

Remark 17 In the previous case, we construct $\Delta^{0}(w)$ from $\Delta^{2}(w)$, applying $\Delta^{-1}$ twice. We will always proceed this way. 
Case (2) If $p=a a b$, then

$$
\begin{aligned}
\Delta^{0}(w) & =\left(a^{a} b^{a}\right)^{\frac{b-1}{2}} a^{a}\left(b^{b} \underline{a}^{b}\right)^{\frac{b-1}{2}} b^{b} \ldots \\
\Delta^{1}(w) & =a^{b} b^{b} \ldots \\
\Delta^{2}(w) & =b b \cdots
\end{aligned}
$$

$w$ has the factor $f=a^{b}$ smaller than its prefix $a^{a} b^{a}$.

Case (3) If $p=a b a a$, then

$$
\begin{aligned}
\Delta^{0}(w) & =\left(\left(a^{b} b^{b}\right)^{\frac{a}{2}}\left(a^{a} b^{a}\right)^{\frac{a}{2}}\right)^{\frac{a}{2}}\left(\left(\underline{a}^{b} b^{b}\right)^{\frac{b-1}{2}} a^{b}\left(b^{a} a^{a}\right)^{\frac{b-1}{2}} b^{a}\right)^{\frac{a}{2}} \ldots \\
\Delta^{1}(w) & =\left(b^{a} a^{a}\right)^{\frac{a}{2}}\left(b^{b} a^{b}\right)^{\frac{a}{2}} \ldots \\
\Delta^{2}(w) & =a^{a} b^{a} \cdots \\
\Delta^{3}(w) & =a a \cdots
\end{aligned}
$$

$w$ has the factor $f=\left(a^{b} b^{b}\right)^{\frac{b-1}{2}} a^{b}$.

Case (4) If $p=a b a b$, then

$$
\begin{aligned}
\Delta^{0}(w) & =\left(\left(a^{b} b^{b}\right)^{\frac{a}{2}}\left(a^{a} b^{a}\right)^{\frac{a}{2}}\right)^{\frac{b-1}{2}}\left(a^{b} b^{b}\right)^{\frac{a}{2}}\left(\left(a^{a} b^{a}\right)^{\frac{b-1}{2}} a^{a}\left(b^{b} a^{b}\right)^{\frac{b-1}{2}} b^{b}\right)^{\frac{b-1}{2}}\left(a^{a} b^{a}\right)^{\frac{b-1}{2}} a^{a}\left(b^{b} \underline{a}^{b}\right)^{\frac{a}{2}} b^{a} a \cdots \\
\Delta^{1}(w) & =\left(b^{a} a^{a}\right)^{\frac{b-1}{2}} b^{a}\left(a^{b} b^{b}\right)^{\frac{b-1}{2}} a^{b} b^{a} a \cdots \\
\Delta^{2}(w) & =a^{b} b^{b} a \cdots \\
\Delta^{3}(w) & =b b \cdots
\end{aligned}
$$

$w$ has the factor $f=a^{b} b^{a} a$.

Remark 18 In the computation of $\Phi^{-1}(a b a b)$, we deduce the last letter of each line, as we did in Example 9. This deduction will be used in further cases.

Case (5) If $p=a b b$, then

$$
\begin{aligned}
\Delta^{0}(w) & =\left(a^{b} b^{b}\right)^{\frac{b-1}{2}} \underline{a}^{b}\left(b^{a} a^{a}\right)^{\frac{b-1}{2}} b^{a} \ldots \\
\Delta^{1}(w) & =b^{b} a^{b} \cdots \\
\Delta^{2}(w) & =b b \cdots
\end{aligned}
$$

$w$ has the factor $f=a^{b} b^{a} a^{a}$.

We conclude using Proposition 13 ii). 


\subsection{Over an even alphabet}

Let us now consider the case of an alphabet $\mathcal{A}$ having even letters.

Theorem 19 Over the alphabet $\{a<b\}$, with a and $b$ even, the only infinite smooth word that is also a Lyndon word is $m_{\{a<b\}}$.

Proof: We proceed similarly as in the previous subsection. The four possibilities are illustrated in Figure 2. We will first prove that Cases (1) and (2) are impossible and then, we will show in Case (3) that $a b b$ is a prefix of an infinite smooth Lyndon word $w$ if and only if $w=\Phi^{-1}\left(a b^{\omega}\right)=m_{\{a, b\}}$.

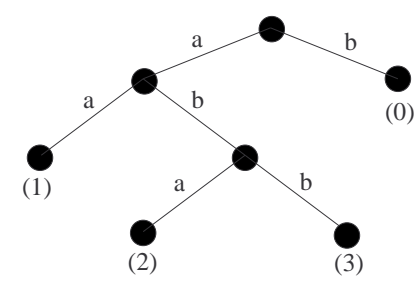

Figure 2: Possible cases for an even alphabet

Case (1) If $p=a a x$, with $x \in \mathcal{A}$, then

$$
\begin{aligned}
\Delta^{0}(w) & =\left(a^{a} b^{a}\right)^{\frac{x}{2}}\left(\underline{a}^{b} b^{b}\right)^{\frac{x}{2}} \ldots \\
\Delta^{1}(w) & =a^{x} b^{x} \ldots \\
\Delta^{2}(w) & =x x \cdots
\end{aligned}
$$

$w$ has the factor $f=a^{b}$.

Case (2) If $p=a b a x$, with $x \in \mathcal{A}$, then

$$
\begin{aligned}
\Delta^{0}(w) & =\left(\left(a^{b} b^{b}\right)^{\frac{a}{2}}\left(a^{a} b^{a}\right)^{\frac{a}{2}}\right)^{\frac{x}{2}}\left(\left(\underline{a}^{b} b^{b}\right)^{\frac{b}{2}}\left(a^{a} b^{a}\right)^{\frac{b}{2}}\right)^{\frac{x}{2}} \ldots \\
\Delta^{1}(w) & =\left(b^{a} a^{a}\right)^{\frac{x}{2}}\left(b^{b} a^{b}\right)^{\frac{x}{2}} \cdots \\
\Delta^{2}(w) & =a^{x} b^{x} \cdots \\
\Delta^{3}(w) & =x x \cdots
\end{aligned}
$$

$w$ has the factor $f=\left(a^{b} b^{b}\right)^{\frac{b}{2}}$.

Case (3) Recall that the minimal infinite smooth word $m_{\{a, b\}}=\Phi^{-1}\left(a b^{\omega}\right)$ is a Lyndon word. Let us show that for an even alphabet, it is the only smooth word that is also a Lyndon word. In order to prove it, let us suppose that we can write $p=a b^{k} a y$, with $k \geq 2$ maximal, since Case (2) already excluded the possibility $k=1$, and $y \in \mathcal{A}^{\omega}$. Let us first compute $u=\Phi^{-1}$ (bbay), with 


$$
y[0]=x \in \mathcal{A} \text {. We get }
$$

$$
\begin{aligned}
\Delta^{0}(u) & =\left(\left(b^{b} a^{b}\right)^{\frac{a}{2}}\left(b^{a} a^{a}\right)^{\frac{a}{2}}\right)^{\frac{x}{2}}\left(\left(b^{b} a^{b}\right)^{\frac{b}{2}}\left(b^{a} a^{a}\right)^{\frac{b}{2}}\right)^{\frac{x}{2}} \ldots \\
\Delta^{1}(u) & =\left(b^{a} a^{a}\right)^{\frac{x}{2}}\left(b^{b} a^{b}\right)^{\frac{x}{2}} \ldots \\
\Delta^{2}(u) & =a^{x} b^{x} \cdots \\
\Delta^{3}(u) & =x x \cdots
\end{aligned}
$$

Since $a$ and $b$ are even and using Lemma $12, \Phi^{-1}\left(b^{k} a y\right)$ can be then written as

$$
\left(v_{1}^{\frac{a}{2}} v_{2}^{\frac{a}{2}}\right)^{\frac{x}{2}}\left(v_{1}^{\frac{b}{2}} v_{2}^{\frac{b}{2}}\right)^{\frac{x}{2}} s
$$

with $v_{1}=\Delta_{b}^{-(k-2)}\left(b^{b} a^{b}\right), v_{2}=\Delta_{b}^{-(k-2)}\left(b^{a} a^{a}\right), s \in \mathcal{A}^{\omega}$ and $x \in \mathcal{A}$.

Moreover, since $\Phi^{-1}\left(b^{\omega}\right)$ is the maximal infinite smooth word and since $\Phi^{-1}\left(b^{k}\right)$ (resp. $\Phi^{-1}\left(b^{k-1} a\right)$ ) is a prefix of $v_{1}$ (resp. $v_{2}$ ), we have that $v_{1}>v_{2}$ and $v_{1}$ is not a prefix of $v_{2}$. Furthermore for $k \geq 2$, using Equation (1) we get

$$
\Delta_{b}^{-1}\left(v_{1}\right)>\Delta_{b}^{-1}\left(v_{2}\right) \Longleftrightarrow \Delta_{a}^{-1}\left(v_{1}\right)<\Delta_{a}^{-1}\left(v_{2}\right)
$$

that implies

$$
\Delta_{a}^{-1}\left(v_{1}^{\frac{b}{2}}\right)<\Delta_{a}^{-1}\left(v_{1}^{\frac{a}{2}} v_{2}^{\frac{a}{2}}\right)
$$

Thus $\Phi^{-1}\left(a b^{k} a y\right)$ has the prefix $\Delta_{a}^{-1}\left(v_{1}^{\frac{a}{2}} v_{2}^{\frac{a}{2}}\right)$ and the smaller factor that is not a prefix, $\Delta_{a}^{-1}\left(v_{1}^{\frac{b}{2}}\right)$. Consequently, $\Phi^{-1}\left(a b^{k} a y\right)$ is not a Lyndon word. Finally, we conclude that $\Phi^{-1}\left(a b b p^{\prime}\right)$ is an infinite smooth Lyndon word if and only if $p^{\prime}=b^{\omega}$.

The only smooth Lyndon word over an even two-letter alphabet is the minimal smooth word $m_{\mathcal{A}}$ with $\Phi\left(m_{\mathcal{A}}\right)=a b^{\omega}$.

\subsection{Over an odd alphabet}

In this section, we prove the following result.

Theorem 20 Over the alphabet $\{a<b\}$, with a and $b$ odd, there exist two infinite smooth Lyndon words if and only if $a=1$. More precisely, the infinite smooth Lyndon words are the minimal smooth word $m_{\{1<b\}}$ and $\Delta_{1}^{-1}\left(m_{\{1, b\}}\right)$.

Before proving Theorem 20, some results are required.

Lemma 21 Let $\mathcal{A}=\{a<b\}$ be an odd alphabet. Let $w, w^{\prime}$ be two finite factors of an infinite smooth word such that $w<w^{\prime}$ and $w=x a y, w^{\prime}=x b y^{\prime}$, with $x, y, y^{\prime} \in \mathcal{A}^{*}$. If $|x|$ is even (resp. odd), then

$$
\Delta_{\alpha}^{-1}(w)<\Delta_{\alpha}^{-1}\left(w^{\prime}\right) \Longleftrightarrow \bar{\alpha}<\alpha(\text { resp. } \alpha<\bar{\alpha})
$$

with $\alpha \in \mathcal{A}$ and $\bar{\alpha}$ its complement. 
Proof: Assume $|x|$ even. Then $\Delta_{\alpha}^{-1}(x)$ ends by $\bar{\alpha}$. By direct computation, we have the following equalities:

$$
\Delta_{\alpha}^{-1}(w)=\Delta_{\alpha}^{-1}(x a y) \quad=\Delta_{\alpha}^{-1}(x) \Delta_{\alpha}^{-1}(a) \Delta_{\bar{\alpha}}^{-1}(y)=\Delta_{\alpha}^{-1}(x) \alpha^{a} \Delta_{\bar{\alpha}}^{-1}(y)
$$

and

$$
\Delta_{\alpha}^{-1}\left(w^{\prime}\right)=\Delta_{\alpha}^{-1}\left(x b y^{\prime}\right) \quad=\Delta_{\alpha}^{-1}(x) \Delta_{\alpha}^{-1}(b) \Delta_{\alpha}^{-1}\left(y^{\prime}\right)=\Delta_{\alpha}^{-1}(x) \alpha^{b} \Delta_{\bar{\alpha}}^{-1}\left(y^{\prime}\right) .
$$

Then $\Delta_{\alpha}^{-1}(w)<\Delta_{\alpha}^{-1}\left(w^{\prime}\right)$ if and only if $\Delta_{\bar{\alpha}}^{-1}(y)[0]<\alpha$. We conclude since $\Delta_{\bar{\alpha}}^{-1}(y)[0]=\bar{\alpha}$. A similar argument holds for $|x|$ odd.

Now, let us prove two subcases of Theorem 20: $a \neq 1$ (Theorem 22) and $a=1$ (Theorem 29).

Theorem 22 Over the alphabet $\{a<b\}$, with $a, b$ odd and $a \neq 1$, there is no infinite smooth word that is also a Lyndon word.

Proof: As in Sections 3.1 and 3.2, we proceed by inspection of the different possible prefixes of $\Phi(w)$ (see Figure 3) for an infinite smooth word $w$.

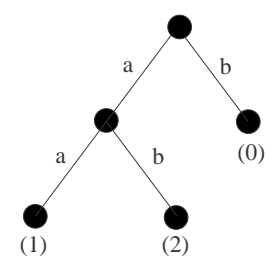

Figure 3: Possible cases for an odd alphabet, with $a \neq 1$

Case (1) If $p=a a x$, with $x \in \mathcal{A}$, then

$$
\begin{aligned}
\Delta^{0}(w) & =\left(a^{a} b^{a}\right)^{\frac{x-1}{2}} a^{a}\left(b^{b} \underline{a}^{b}\right)^{\frac{x-1}{2}} b^{b}\left(a^{a} b^{a}\right)^{\frac{x-1}{2}} a^{a} \cdots \\
\Delta^{1}(w) & =a^{x} b^{x} a^{x} \cdots \\
\Delta^{2}(w) & =x x x \cdots
\end{aligned}
$$

Since $1<a \leq x$ and $x$ is odd, $x \geq 3$ and then, $\frac{x-1}{2} \geq 1$. Thus, $w$ has the factor $f=a^{b}$.

Remark 23 For the same reasons as in Remark 16, we can assume that $\Delta^{2}(w)$ starts by a block of length at least 3 .

Case (2) If $p=a b x$, with $x \in \mathcal{A}$, then

$$
\begin{aligned}
\Delta^{0}(w) & =\left(a^{b} b^{b}\right)^{\frac{x-1}{2}} \underline{a}^{b}\left(b^{a} a^{a}\right)^{\frac{x-1}{2}} b^{a}\left(a^{b} b^{b}\right)^{\frac{x-1}{2}} a^{b} \cdots \\
\Delta^{1}(w) & =b^{x} a^{x} b^{x} \cdots \\
\Delta^{2}(w) & =x x x \cdots
\end{aligned}
$$

$w$ has the factor $f=a^{b} b^{a} a^{a}$. 
Since in the 3 cases, it is possible to find a factor smaller than the prefix, we conclude that there is no smooth Lyndon word over an odd alphabet $\mathcal{A}$, with $a \neq 1$.

Lemma 24 Let $w$ be an infinite smooth word over an odd alphabet $\{a<b\}$, starting by the letter a (resp. b). Then, a block of a's always starts in an even (resp. odd) position and a block of b's always starts in an odd (resp. even) position.

Proof: It follows from the parity of the letters of the alphabet: every block has odd length, thus between 2 blocks there is an even number of positions.

Lemma 25 In the infinite smooth word $w=\Phi^{-1}\left((1 b)^{\omega}\right)$, the blocks of $b^{\prime} s$ have all length 1.

Proof: It is already proved for $b=3$ in [BMP07] and follows from the parity of the letters and the alternation of the letters in $\Phi(w)$.

We know from [BJP08] that the minimal infinite smooth word over an odd two-letter alphabet $\{1<b\}$ is a Lyndon word. The next proposition shows that this is the only infinite Lyndon word $w$ over the alphabet $\{1<b\}$ such that $\Phi(w)$ starts by $1 b$.

Proposition 26 Over the alphabet $\{1<b\}$, with $b$ odd, the only infinite smooth Lyndon word $w$ such that $\Phi(w)$ starts by $1 b$ is $m_{\{1<b\}}$.

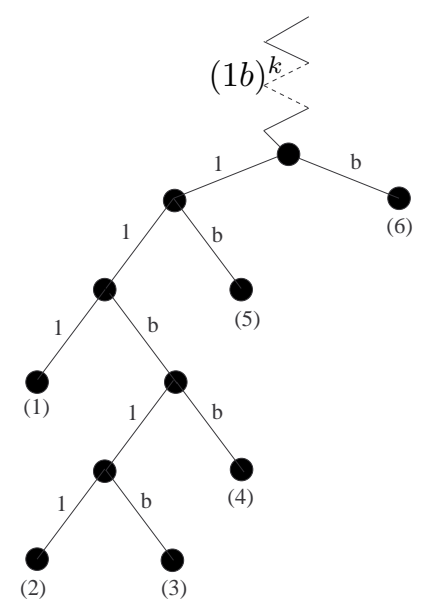

Figure 4: Possible prefixes of $p$ starting by $(1 b)^{k}, k \geq 1$

Proof: Recall first that $\Phi\left(m_{\{1, b\}}\right)=(1 b)^{\omega}$. Let us consider all possible prefixes $p$ such that $\Phi^{-1}(p)$ is a prefix of an infinite smooth Lyndon word. Since we suppose that $p$ starts by $1 b$, let us rewrite $p$ as $(1 b)^{k} s$, where $k$ is maximal and $s \in \mathcal{A}^{\omega}$. We then proceed by inspection of the different possibilities (see Figure 4). 
Case (1) If $p=(1 b)^{k} 111$, let us consider the prefix $\Phi^{-1}(1 b 111)$ of $u$.

$$
\begin{aligned}
\Delta^{0}(u) & =1^{b}\left((b 1)^{\frac{b-1}{2}} b\left(1^{b} b^{b}\right)^{\frac{b-1}{2}} \underline{1}^{b}\right)^{\frac{b-1}{2}}(b 1)^{\frac{b-1}{2}} b 1^{b} b 1 \cdots \\
\Delta^{1}(u) & =b\left(1^{b} b^{b}\right)^{\frac{b-1}{2}} 1^{b} b 1 \cdots \\
\Delta^{2}(u) & =1 b^{b} 1 \cdots \\
\Delta^{3}(u) & =1 b \cdots \\
\Delta^{4}(u) & =1 \cdots
\end{aligned}
$$

$u$ has the factor $f=1^{b}(b 1)^{\frac{b-1}{2}} b 1^{b} b 1$. Using Lemma $212(k-1)$ times, Lemmas 24 and 25 , we conclude that the prefix $p$ does not describe a prefix of a Lyndon word.

Case (2) If $p=(1 b)^{k} 11 b 11$, let us consider the prefix $\Phi^{-1}(1 b 11 b 11)$ of $u$.

$$
\begin{aligned}
\Delta^{0}(u)= & \left(1^{b}(b 1)^{\frac{b-1}{2}} b\right)^{\frac{b-1}{2}} 1^{b}\left(\left((b 1)^{\frac{b-1}{2}} b\left(1^{b} b^{b}\right)^{\frac{b-1}{2}} 1^{b}\right)^{\frac{b-1}{2}}(b 1)^{\frac{b-1}{2}} b 1^{b}\right)^{\frac{b-1}{2}}\left(b 1^{b}\right)^{\frac{b-1}{2}} b\left(\left(1^{b} b^{b}\right)^{\frac{b-1}{2}} 1^{b} b\right)^{\frac{b-1}{2}} \\
& \left(1^{b} b^{b}\right)^{\frac{b-1}{2}} 1^{b}\left(b 1^{b}\right)^{\frac{b-1}{2}} b\left(1^{b} b^{b}\right)^{\frac{b-1}{2}} 1^{b} b 1 \cdots \\
\Delta^{1}(u)= & \left(b 1^{b}\right)^{\frac{b-1}{2}} b\left(\left(1^{b} b^{b}\right)^{\frac{b-1}{2}} 1^{b} b\right)^{\frac{b-1}{2}}(1 b)^{\frac{b-1}{2}} 1\left(b^{b} 1\right)^{\frac{b-1}{2}} b^{b}(1 b)^{\frac{b-1}{2}} 1 b^{b} 1 \cdots \\
\Delta^{2}(u)= & (1 b)^{\frac{b-1}{2}} 1\left(b^{b} 1\right)^{\frac{b-1}{2}} 1^{b}(b 1)^{\frac{b-1}{2}} b 1^{b} b \cdots \\
\Delta^{3}(u)= & 1^{b}(b 1)^{\frac{b-1}{2}} b 1^{b} b \cdots \\
\Delta^{4}(u)= & b 1^{b} b \cdots \\
\Delta^{5}(u)= & 1 b \cdots \\
\Delta^{6}(u)= & 1 \cdots
\end{aligned}
$$

$u$ has the factor $f=1^{b} b\left(1^{b} b^{b}\right)^{\frac{b-1}{2}}$. Applying Lemma $212(k-1)$ times and using Lemmas 24 and 25 , we conclude.

Case (3) If $p=(1 b)^{k} 11 b 1 b$, let us consider the prefix $\Phi^{-1}(11 b 1 b)$ of $u$.

$$
\begin{aligned}
\Delta^{0}(u) & =\left((1 b)^{\frac{b-1}{2}} 1 b^{b}\right)^{\frac{b-1}{2}}(1 b)^{\frac{b-1}{2}} 1\left(b^{b} 1\right)^{\frac{b-1}{2}} \underline{1}^{b}(b 1)^{\frac{b-1}{2}} b 1^{b} b \ldots \\
\Delta^{1}(u) & =\left(1^{b} b\right)^{\frac{b-1}{2}} 1^{b}(b 1)^{\frac{b-1}{2}} b 1^{b} b \ldots \\
\Delta^{2}(u) & =(b 1)^{\frac{b-1}{2}} b 1^{b} b \ldots \\
\Delta^{3}(u) & =1^{b} b \ldots \\
\Delta^{4}(u) & =b \cdots
\end{aligned}
$$

$u$ has the factor $f=1^{b}$. Applying Lemma $21(2 k)$ times and using Lemmas 24 and 25, we conclude. 
Case (4) If $p=(1 b)^{k} 11 b b$, let us consider the prefix $\Phi^{-1}(1 b 11 b b)$ of $u$.

$$
\begin{aligned}
& \Delta^{0}(u)=\left(\left(1^{b}(b 1)^{\frac{b-1}{2}} b\right)^{\frac{b-1}{2}} 1^{b}\left(\left((b 1)^{\frac{b-1}{2}} b\left(1^{b} b^{b}\right)^{\frac{b-1}{2}} 1^{b}\right)^{\frac{b-1}{2}}(b 1)^{\frac{b-1}{2}} b\left(1^{b} b\right)^{\frac{b-1}{2}} 1^{b}\right)^{\frac{b-1}{2}} \ldots\right. \\
& \Delta^{1}(u)=\left(\left(b 1^{b}\right)^{\frac{b-1}{2}} b\left(\left(1^{b} b^{b}\right)^{\frac{b-1}{2}} 1^{b}(b 1)^{\frac{b-1}{2}} b\right)^{\frac{b-1}{2}}\left(1^{b} b^{b}\right)^{\frac{b-1}{2}} 1^{b}\right)^{\frac{b-1}{2}}\left(b 1^{b}\right)^{\frac{b-1}{2}} b\left(1^{b} b^{b}\right)^{\frac{b-1}{2}} 1^{b} b 1^{b} b \cdots \\
& \Delta^{2}(u)=\left((1 b)^{\frac{b-1}{2}} 1\left(b^{b} 1^{b}\right)^{\frac{b-1}{2}} b^{b}\right)^{\frac{b-1}{2}}(1 b)^{\frac{b-1}{2}} 1 b^{b} 1 b \cdots \\
& \Delta^{3}(u)=\left(1^{b} b^{b}\right)^{\frac{b-1}{2}} 1^{b} b 1 \ldots \\
& \Delta^{4}(u)=b^{b} 1 \cdots \\
& \Delta^{5}(u)=b \cdots
\end{aligned}
$$

$u$ has the factor $f=1^{b}(b 1)^{\frac{b-1}{2}} b\left(1^{b} b\right)^{\frac{b-1}{2}}$. Applying Lemma $212(k-1)$ times and using Lemmas 24 and 25, we conclude.

Case (5) If $p=(1 b)^{k}(1 b)$, it is a prefix of $m_{\{1, b\}}$, which is a smooth Lyndon word.

Case (6) If $p=(1 b)^{k} b$, let us consider $v=\Phi^{-1}\left((1 b)^{k} \cdot(b-1)\right)$. This word is illustrated at the left-side of the line in Figure 5. Only the first and the last letters of each line are written. Since 1 and $b$ are odd and $(b-1)$ has even length, the $(2 k-1)$ first lines have even length, following that the first and the last letters differ. The first letter at the right-side of Figure 5 are written. Then using Lemma 12, we get

$$
\Phi^{-1}(p)=\Phi^{-1}\left((1 b)^{k} b\right)=\Phi^{-1}\left((1 b)^{k}(b-1)\right) \Phi^{-1}\left((1 b)^{k} 1\right) .
$$

Since $\Phi^{-1}\left((1 b)^{k} 1\right)$ is a prefix of the minimal smooth word, it is smaller than $\Phi^{-1}\left((1 b)^{k} b\right)$. We conclude that $\Phi^{-1}(p)$ is not a Lyndon word.

Lemma 27 The word $\Delta_{1}^{-1}\left(m_{\{1, b\}}\right)$ is an infinite smooth Lyndon word over the odd alphabet $\{1<b\}$.

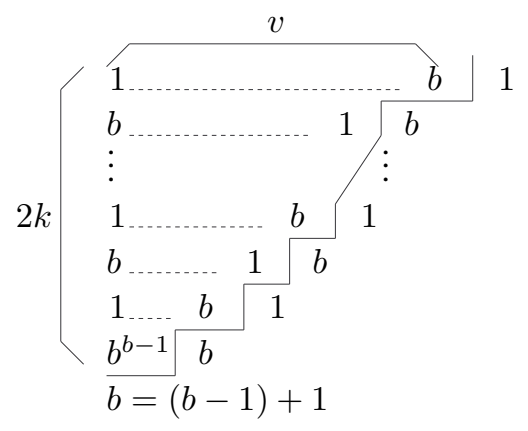

Figure 5: Case (6) 
Proof: By Proposition 11 ii), we know that the minimal infinite smooth word $\Phi^{-1}\left((1 b)^{\omega}\right)$ is a Lyndon word. It is then sufficient to prove that this implies that $\Delta_{1}^{-1}\left(m_{\{1, b\}}\right)$ is also a Lyndon word. Let us consider a factor $f$ of $m_{\{1, b\}}$. Since $m_{\{1, b\}}$ is a Lyndon word, $m_{\{1, b\}}<f$. Then, let us compute $F=\Delta^{-1}(f)$ in $\Delta_{1}^{-1}\left(m_{\{1, b\}}\right)$. There are two cases to consider.

i) If $m_{\{1, b\}}=11 y$ and $f=1 b y^{\prime}$, with $y \in \mathcal{A}^{\omega}, y^{\prime} \in \mathcal{A}^{*}$ : then from Lemma 24, $f[1]$ is in an odd position, implying that $f$ starts in an even position. Thus, $F=\Delta_{1}^{-1}(f)=1 b^{b} \cdots$ and $\Delta_{1}^{-1}\left(m_{\{1, b\}}\right)=1 b 1 \cdots$. Consequently, $\Delta_{1}^{-1}\left(m_{\{1, b\}}\right)<F$.

ii) If $m_{\{1, b\}}=1^{b} x 1 y$ and $s=1^{b} x b y^{\prime}$, with $x, y^{\prime} \in \mathcal{A}^{*}, y \in \mathcal{A}^{\omega}$ : then from Lemma 25, we know that the $b$ in $s$ is at the beginning of a block, so it is at an odd position. Furthermore, by Lemma 24, the block $1^{b}$ starts in an even position. Thus, $1^{b} x$ has odd length. Applying Lemma 21, we conclude that $\Delta_{1}^{-1}\left(m_{\{1, b\}}\right)<F$, and consequently, $\Delta_{1}^{-1}\left(m_{\{1, b\}}\right)$ is an infinite smooth Lyndon word.

Proposition 28 Over the alphabet $\{1<b\}$, with $b$ odd, the only infinite smooth Lyndon word $w$ such that $\Phi(w)$ starts by 11 is $\Delta_{1}^{-1}\left(m_{\{1, b\}}\right)$.

Proof: Let $p$ be a prefix of $\Phi(w)$. By Lemma 14, we have $p[0]=1$. Let us prove that the only infinite smooth Lyndon word $w$ such that $p$ starts by 11 is $\Delta_{1}^{-1}\left(m_{\{1, b\}}\right)$. We proceed by inspection of the different cases illustrated in Figure 6.

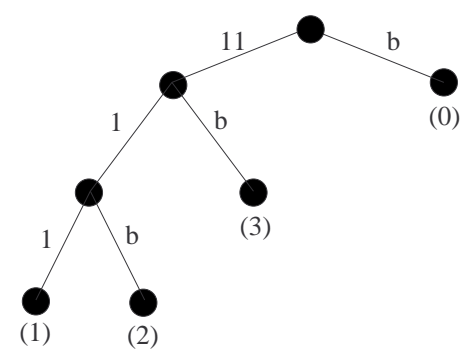

Figure 6: Possible prefixes of $p$ starting by 11

Case (1) If $p=1111$, then

$$
\begin{aligned}
\Delta^{0}(w) & =1\left(b^{b} \underline{1}^{b}\right)^{\frac{b-1}{2}} b^{b} 1 b \cdots \\
\Delta^{1}(w) & =1 b^{b} 1 \cdots \\
\Delta^{2}(w) & =1 b \cdots \\
\Delta^{3}(w) & =1 \cdots
\end{aligned}
$$

$w$ has the factor $1^{b}$. 
Case (2) If $p=111 b$, then

$$
\begin{aligned}
\Delta^{0}(w) & =\left(1 b^{b}\right)^{\frac{b-1}{2}} 1\left(b^{b} \underline{1}^{b}\right)^{\frac{b-1}{2}} b^{b} 1 \cdots \\
\Delta^{1}(w) & =(1 b)^{\frac{b-1}{2}} 1 b^{b} 1 \cdots \\
\Delta^{2}(w) & =1^{b} b \cdots \\
\Delta^{3}(w) & =b \cdots
\end{aligned}
$$

$w$ has the factor $1^{b}$

Case (3) If $p=11 b$, then let us prove that the only smooth Lyndon word $w$ having $p$ has a prefix of $\Phi(w)$ is $\Delta_{1}^{-1}\left(m_{\{1, b\}}\right)$. To do so, we will prove that $\Phi^{-1}(11 b s)$ is a Lyndon word if and only if $\Phi^{-1}(1 b s)$ is so. Let us consider $w=\Phi^{-1}(1 b s)$ and $f$, a factor of $w$. Let us suppose that $w$ is not a Lyndon word and let us show that it implies that $\Delta_{1}^{-1}(w)$ is not too. Let us rewrite $w=1^{b} x b y$ and $f=1^{b} x 1 y^{\prime}$, with $x, y^{\prime} \in \mathcal{A}^{*}$ and $y \in \mathcal{A}^{\omega}$. There are two cases to consider.

i) If $x$ ends by $b$ : rewrite $f=1^{b} x^{\prime} b 1 y^{\prime}$ and $w=1^{b} x^{\prime} b b y$. Then, $w$ contains the factor $b^{b}$. The existence of this factor in $w$ implies that there is a factor $1^{b}$ in the word $\Delta_{1}^{-1}(w)$. Consequently, since $\Delta_{1}^{-1}(w)$ starts by $1 b$, it cannot be a Lyndon word.

ii) If $x$ ends by 1 : rewrite $f=1^{b} x^{\prime} 11 y^{\prime}$ and $w=1^{b} x^{\prime} 1 b y$. By Lemma $24, f$ starts in an even position. Then let us compute $\Delta_{1}^{-1}(f)$ and $\Delta_{1}^{-1}(w)$. Again, by Lemma 24, we have that the $b$ in $w$ is in an odd position, implying that $1^{b} x^{\prime} 1$ has odd length. Using Lemma 21 , we conclude that $f<w \Rightarrow \Delta_{1}^{-1}(f)<\Delta_{1}^{-1}(w): \Delta_{1}^{-1}(w)$ is not a Lyndon word.

We conclude, using Lemma 27.

Theorem 29 Over the alphabet $\{1<b\}$, with $b$ odd, the only infinite smooth words that are also Lyndon words are $m_{\{1<b\}}$ and $\Delta_{1}^{-1} m_{\{1<b\}}$.

Proof: Follows from the union of Propositions 26 and 28.

Proof: (of Theorem 20) Follows from Theorems 22 and 29.

\subsection{Over $\mathcal{A}$ with $a$ odd and $b$ even}

In this section, we consider infinite smooth words over an alphabet $\{a<b\}$, with $a$ odd and $b$ even. We prove that over this alphabet, there is no infinite smooth word that is also a Lyndon word. In order to prove it, we consider two cases that have to be analyzed separately: $a \neq 1$ and $a=1$.

Theorem 30 Over the alphabet $\{a<b\}$, with $a \neq 1$ odd and $b$ even, there is no smooth infinite word that is a Lyndon word.

Proof: There are 5 possibilities to consider, illustrated in Figure 7 (a). 


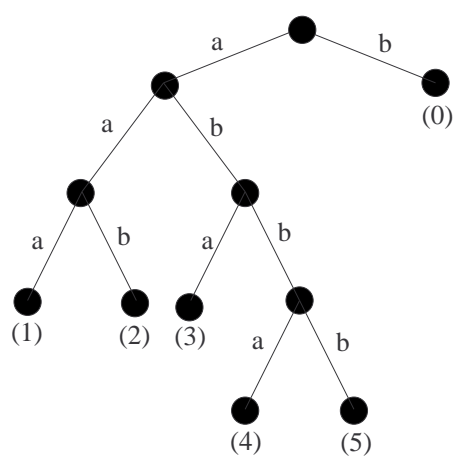

(a) for an odd-even alphabet $\{a<b\}$, with $a \neq 1$.

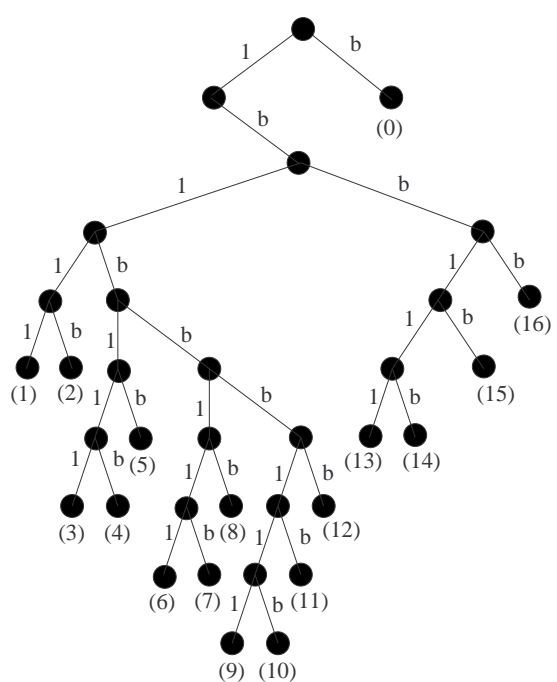

(b) for the alphabet $\{a<b\}$, with $a=1$ and $b=4 n$.

Figure 7: Possible cases

Case (1) If $p=a a a$, then,

$$
\begin{aligned}
\Delta^{0}(w) & =\left(a^{a} b^{a}\right)^{\frac{a-1}{2}} a^{a}\left(b^{b} \underline{a}^{b}\right)^{\frac{a-1}{2}} b^{b} \ldots \\
\Delta^{1}(w) & =a^{a} b^{a} \ldots \\
\Delta^{2}(w) & =a a \cdots
\end{aligned}
$$

$w$ has the factor $a^{b}$.

Case (2) If $p=a a b$, then,

$$
\begin{aligned}
\Delta^{0}(w) & =\left(a^{a} b^{a}\right)^{\frac{b}{2}}\left(\underline{a}^{b} b^{b}\right)^{\frac{b}{2}} \cdots \\
\Delta^{1}(w) & =a^{b} b^{b} \ldots \\
\Delta^{2}(w) & =b b \cdots
\end{aligned}
$$

$w$ has the factor $a^{b}$.

Case (3) If $p=a b a$, then,

$$
\begin{aligned}
\Delta^{0}(w) & =\left(a^{b} b^{b}\right)^{\frac{a-1}{2}} \underline{a}^{b}\left(b^{a} a^{a}\right)^{\frac{a-1}{2}} b^{a} \cdots \\
\Delta^{1}(w) & =b^{a} a^{a} \cdots \\
\Delta^{2}(w) & =a a \cdots
\end{aligned}
$$

$w$ has the factor $a^{b} b^{a} a^{a}$. 
Case (4) If $p=a b b a$, then,

$$
\begin{aligned}
\Delta^{0}(w)= & \left(\left(a^{b} b^{b}\right)^{\frac{b}{2}}\left(a^{a} b^{a}\right)^{\frac{b}{2}}\right)^{\frac{a-1}{2}}\left(a^{b} b^{b}\right)^{\frac{b}{2}}\left(\left(a^{a} b^{a}\right)^{\frac{a-1}{2}} a^{a}\left(b^{b} a^{b}\right)^{\frac{a-1}{2}} b^{b}\right)^{\frac{a-1}{2}}\left(a^{a} b^{a}\right)^{\frac{a-1}{2}} a^{a} \\
& \left(\left(b^{b} \underline{a}^{b}\right)^{\frac{b}{2}}\left(b^{a} a^{a}\right)^{\frac{b}{2}}\right)^{\frac{a-1}{2}}\left(b^{b} a^{b}\right)^{\frac{b}{2}} \ldots \\
\Delta^{1}(w)= & \left(b^{b} a^{b}\right)^{\frac{a-1}{2}} b^{b}\left(a^{a} b^{a}\right)^{\frac{a-1}{2}} a^{a}\left(b^{b} a^{b}\right)^{\frac{a-1}{2}} b^{b} \ldots \\
\Delta^{2}(w)= & b^{a} a^{a} b^{a} \ldots \\
\Delta^{3}(w)= & a a a
\end{aligned}
$$

$w$ has the factor $a^{b} b^{a} a^{a}$.

Case (5) If $p=a b b b$, then,

$$
\begin{aligned}
\Delta^{0}(w) & =\left(\left(a^{b} b^{b}\right)^{\frac{b}{2}}\left(a^{a} b^{a}\right)^{\frac{b}{2}}\right)^{\frac{b}{2}}\left(\left(a^{b} b^{b}\right)^{\frac{a-1}{2}} \underline{a}^{b}\left(b^{a} a^{a}\right)^{\frac{a-1}{2}} b^{a}\right)^{\frac{b}{2}} \ldots \\
\Delta^{1}(w) & =\left(b^{b} a^{b}\right)^{\frac{b}{2}}\left(b^{a} a^{a}\right)^{\frac{b}{2}} \ldots \\
\Delta^{2}(w) & =b^{b} a^{b} \cdots \\
\Delta^{3}(w) & =b b \cdots
\end{aligned}
$$

$w$ has the factor $a^{b} b^{a} a^{a}$.

In each case, it is possible to find a factor $f$ smaller than the smooth word $w$. Thus, there is no smooth Lyndon word.

Lemma 31 Let $w$ be an infinite smooth Lyndon word over the alphabet $\{1<b\}$, with $b$ even. Let $p$ be a prefix of $\Phi(w)$. Then $p[0,1]=1 b$.

Proof: There are four cases to consider, illustrated in Figure 8. We know from Lemma 14 that the Case (0) is excluded. For the three other cases, we show that $1^{b}$ is factor of $w$, so it is not a smooth Lyndon word.

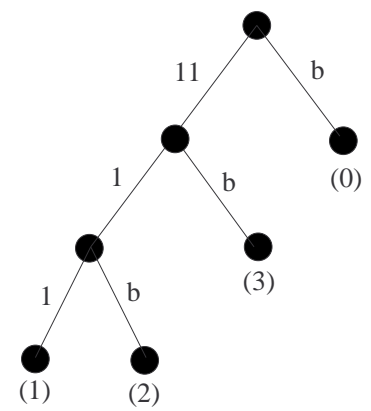

Figure 8: Possible cases. 
Case (1) If $p=1111$, then,

$$
\begin{aligned}
\Delta^{0}(w) & =1\left(b^{b} \underline{1}^{b}\right)^{\frac{b}{2}} b 1 \cdots \\
\Delta^{1}(w) & =1 b^{b} 1 \cdots \\
\Delta^{2}(w) & =1 b \cdots \\
\Delta^{3}(w) & =1 \cdots
\end{aligned}
$$

Case (2) If $p=111 b$, then,

$$
\begin{aligned}
\Delta^{0}(w) & =\left(1 b^{b}\right)^{\frac{b}{2}}(1 b)^{\frac{b}{2}} \underline{1}^{b} b \cdots \\
\Delta^{1}(w) & =(1 b)^{\frac{b}{2}} 1^{b} b \cdots \\
\Delta^{2}(w) & =1^{b} b \cdots \\
\Delta^{3}(w) & =b \cdots
\end{aligned}
$$

Case (3) If $p=11 b$, then,

$$
\begin{aligned}
\Delta^{0}(w) & =(1 b)^{\frac{b}{2}} \underline{1}^{b} b \cdots \\
\Delta^{1}(w) & =1^{b} b \cdots \\
\Delta^{2}(w) & =b \cdots
\end{aligned}
$$

Theorem 32 Over the alphabet $\{a<b\}$, with $a=1$ and $b=4 n$, there is no infinite smooth word that is a Lyndon word.

Proof: Figure 7 (b) shows the different cases to consider. Lemma 31 is used to eliminate the case of a prefix $p$ starting by 11 . For each of the 16 cases, it is again possible to find a factor of $\Phi^{-1}(p)$ in order to prove that it is not a prefix of an infinite smooth Lyndon word. The details are given in Appendix A.

Theorem 33 Over the alphabet $\{a<b\}$, with $a=1$ and $b=2(2 n+1)$, there is no infinite smooth word that is a Lyndon word.

Proof: Figure 9 shows the different cases to consider. Cases numbered less or equal to 16 are the same as in Theorem 32. For the other cases, it is possible to find a factor in $\Phi^{-1}(p)$ smaller than its prefix, implying that the word is not a Lyndon word. The details are given in Appendix B. Again, we use Lemma 31 to eliminate the case of a prefix $p$ starting by 11 .

\section{Summary and concluding remarks}

The next theorem summarizes the results of Section 3.

Theorem 34 Over any two-letter alphabet, the only infinite smooth words that are also infinite Lyndon words are $m_{\{2 a<2 b\}}, m_{\{1<2 b+1\}}$ and $\Delta^{-1}\left(m_{\{1<2 b+1\}}\right)$, for $a, b \in \mathbb{N} \backslash\{0\}$. 


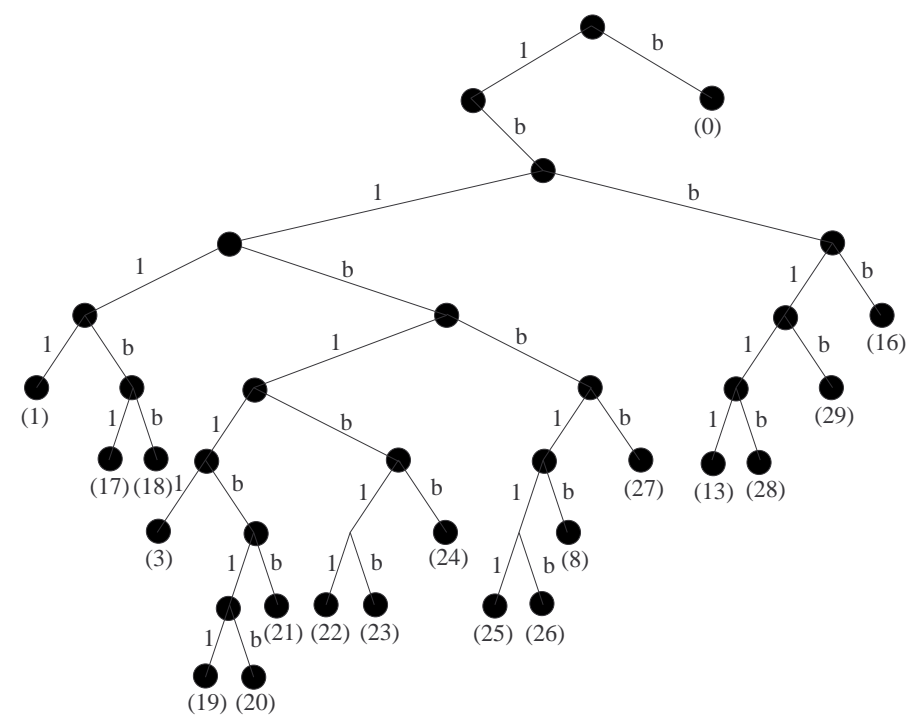

Figure 9: Different cases for the alphabet $\{a<b\}$, with $a=1$ and $b=2(2 n+1)$

Recall that for the alphabet $\{1,2\}$, it is conjectured in [BDLV06] that any smooth factor appears in any infinite smooth word. A direct corollary of this conjecture is that no infinite smooth word is a Lyndon word: there exists a minimal suffix (see [BMP07]), even smaller than the minimal smooth word and a prefix of this suffix must appear in any smooth word. Since we proved in this paper that there is no smooth Lyndon word over the alphabet $\{1,2\}$, our result reinforce the conjecture of [BDLV06].

The existence of infinite smooth Lyndon words over the alphabets $\{2 a<2 b\}$ and $\{1<2 b+1\}$ leads to the following corollary.

Corollary 35 Let $\mathcal{A}$ be a two-letter alphabet such that $\mathcal{A}=\{2 a<2 b\}$ or $\mathcal{A}=\{1<2 b+1\}$. Then, any infinite smooth words $w \in \mathcal{A}^{\omega}$ does not contain every smooth factors.

It is also interesting to notice that our main result completely characterized the trivial finite Lyndon factorization of infinite smooth words: the only infinite smooth words that have a finite Lyndon factorization composed of only one factor are $m_{\{2 a<2 b\}}, m_{\{1<2 b+1\}}$ and $\Delta^{-1}\left(m_{\{1<2 b+1\}}\right)$. It is still an open problem to characterized infinite smooth words that have a non-trivial finite Lyndon factorization. Giving an explicit computation of the Lyndon factorization, finite or infinite, of any infinite smooth words, as Melançon did for characteristic Sturmian words [Me100], is still a challenging problem.

Finally, it would be really nice to find a simpler proof of our result.

\section{Acknowledgements}

The author would like to thank Pierre Lalonde for his interesting question during the LaCIM seminar that leads to this paper and Srecko Brlek for his comments. 


\section{References}

[BBBLP03] A. Bergeron-Brlek, S. Brlek, A. Lacasse, and X. Provençal. Patterns in smooth tilings. In Proceedings of WORDS'03, volume 27 of TUCS Gen. Publ., pages 370-381. Turku Cent. Comput. Sci., Turku, 2003.

[BBC05] V. Berthé, S. Brlek, and P. Choquette. Smooth words over arbitrary alphabets. Theoret. Comput. Sci., 341:293-310, 2005.

[BdL97] J. Berstel and A. de Luca. Sturmian words, Lyndon words and trees. Theoret. Comput. Sci., 178(12):171-203, 1997.

[BDLV06] S. Brlek, S. Dulucq, A. Ladouceur, and L. Vuillon. Combinatorial properties of smooth infinite words. Theoret. Comput. Sci., 352:306-317, 2006.

[BJP08] S. Brlek, D. Jamet, and G. Paquin. Smooth words on 2-letter alphabets having same parity. Theoret. Comput. Sci., 393:166-181, 2008.

[BL03] S. Brlek and A. Ladouceur. A note on differentiable palindromes. Theoret. Comput. Sci., 302:167-178, 2003.

[BMP07] S. Brlek, G. Melançon, and G. Paquin. Properties of the extremal infinite smooth words. Discrete Math. Theoret. Comput. Sci., 9(2):33-49 (electronic), 2007.

[Car94] A. Carpi. On repeated factors in $\mathrm{C}^{\infty}$-words. Information Processing Letters, 52:289-294, 1994.

[CFL58] K. T. Chen, R. H. Fox, and R. C. Lyndon. Free differential calculus, IV. The quotient groups of the lower central series. Ann. of Math., 68:81-95, 1958.

[Dek81] F. M. Dekking. On the structure of self generating sequences. Séminaire de théorie des nombres de Bordeaux, exposé 31, 1980-1981.

[Duv83] J.-P. Duval. Factorizing words over an ordered aphabet. Journal of Algorithms, 4:363-381, 1983.

[Duv88] J.-P. Duval. Génération d'une section des classes de conjugaison et arbre de mots de Lyndon de longueur bornée. Theoret. Comput. Sci., 60:255-283, 1988.

[HR03] C. Hohlweg and C. Reutenauer. Lyndon words, permutations and trees. Theoret. Comput. Sci., 307:173178, 2003.

[Hua97] Y. B. Huang. On the condition of powers of $C^{\infty}$-word to be a $C^{\infty}$-word. Applied Mathematics - $A$ Journal of Chinese Universities (Ser. A), 2:243-246, 1997.

[Hua08] Y. B. Huang. On the number of $C^{\infty}$-words of form $\tilde{w} x w$. Theoret. Comput. Sci., 393:280-286, 2008.

[JP05] D. Jamet and G. Paquin. Discrete surfaces and infinite smooth words. In FPSAC'05 - 17th annual International conference on Formal Power Series and Algebraic Combinatorics (Taormina, Italie) June 20-25, 2005.

[Kol65] W. Kolakoski. Self generating runs, Problem 5304. Amer. Math. Monthly, 72:674, 1965.

[Lot83] M. Lothaire. Combinatorics on words. Addison Wesley, Reading MA, 1983.

[Me100] G. Melançon. Lyndon factorization of Sturmian words. Discrete Appl. Math., 210:137-149, 2000.

[MR89] G. Melançon and C. Reutenauer. Lyndon words, free algebras and shuffles. Canad. J. Math., 4:577-591, 1989.

[Paq08a] G. Paquin. Infinite smooth Lyndon words. In PSC'08 - Prague Stringology Conference (Prague, Czech Republic) Septembre 1 - 3, 2008.

[Paq08b] G. Paquin. Mots équilibrés et mots lisses. PhD thesis, Université du Québec à Montréal, 2008.

[Reu07] C. Reutenauer. Mots de Lyndon généralisés. Sém. Lothar. Combin., 54:Art. B54h, 16 pp. (electronic), 2005/07. 
[Reu93] C. Reutenauer. Free Lie algebras, volume 7. London Math. Soc. Monographs New Ser., 1993.

[Ric07] G. Richomme. Conjugacy of morphisms and Lyndon decomposition of standard Sturmian words. Theoret. Comput. Sci., 380(3):393-400, 2007.

[Séé03] P. Séébold. Lyndon factorization of the Prouhet words. Theoret. Comput. Sci., 307:179-197, 2003.

[SMDS94] R. Siromoney, L. Matthew, V. R. Dare, and K. G. Subramanian. Infinite Lyndon words. Information Processing Letters, 50:101-104, 1994.

[Wea89] W. D. Weakley. On the number of $C^{\infty}$-words of each length. J. Combin. Theory Ser. A, 51(1):55-62, 1989.

\section{A Details of the proof of Theorem 32}

Case (1) If $p=1 b 111$, then

$w$ has the factor $1^{b} b 1^{b}$.

Case (2) If $p=1 b 11 b$, then

$$
\begin{aligned}
\Delta^{0}(w) & =1^{b}\left((b 1)^{\frac{b}{2}}\left(b^{b} \underline{1}^{b}\right)^{\frac{b}{2}}\right)^{\frac{b}{2}} b 1^{b} b \cdots \\
\Delta^{1}(w) & =b\left(1^{b} b^{b}\right)^{\frac{b}{2}} 1 b \cdots \\
\Delta^{2}(w) & =1 b^{b} 1 \cdots \\
\Delta^{3}(w) & =1 b \cdots \\
\Delta^{4}(w) & =1 \cdots \\
\Delta^{0}(w) & =\left(1^{b}(b 1)^{\frac{b}{2}} b^{b}(1 b)^{\frac{b}{2}}\right)^{\frac{b}{4}}\left(\underline{1}^{b} b\right)^{\frac{b}{2}}\left(1^{b} b^{b}\right)^{\frac{b}{2}} 1 b \cdots \\
\Delta^{1}(w) & =\left(b 1^{b}\right)^{\frac{b}{2}}(b 1)^{\frac{b}{2}} b^{b} 1 \cdots \\
\Delta^{2}(w) & =(1 b)^{\frac{b}{2}} 1^{b} b \cdots
\end{aligned}
$$$$
\Delta^{3}(w)=1^{b} b \cdots
$$$$
\Delta^{4}(w)=b \cdots
$$

$w$ has the factor $\left(1^{b} b\right)^{\frac{b}{2}} 1^{b}$.

Case (3) If $p=1 b 1 b 111$, then

$$
\begin{aligned}
& \Delta^{0}(w)\left.=\left(1^{b} b\right)^{\frac{b}{2}}\left(\left(1^{b} b^{b}\right)^{\frac{b}{2}} 1\left(b^{b} 1^{b}\right)^{\frac{b}{2}} b\right)^{\frac{b}{4}}\left(\left(\left(1^{b} b^{b}\right)^{\frac{b}{2}}(1 b)^{\frac{b}{2}}\right)^{\frac{b}{2}}\left(1^{b} b\right)^{\frac{b}{2}}\right)^{\frac{b}{2}}\right)^{\frac{b}{2}}\left(1^{b} b^{b}\right)^{\frac{b}{2}}\left(1 b^{b}\right)^{\frac{b}{2}}(1 b)^{\frac{b}{2}} 1^{b} b \cdots \\
& \Delta^{1}(w)=(b 1)^{\frac{b}{2}}\left(\left(b^{b} 1\right)^{\frac{b}{2}}\left(\left(b^{b} 1^{b}\right)^{\frac{b}{2}}(b 1)^{\frac{b}{2}}\right)^{\frac{b}{2}}\right)^{\frac{b}{2}} b^{b}(1 b)^{\frac{b}{2}} 1^{b} b \cdots \\
& \Delta^{2}(w)=1^{b}\left((b 1)^{\frac{b}{2}}\left(b^{b} 1^{b}\right)^{\frac{b}{2}}\right)^{\frac{b}{2}} b 1^{b} b \cdots \\
& \Delta^{3}(w)=b\left(1^{b} b^{b}\right)^{\frac{b}{2}} 1 b \cdots \\
& \Delta^{4}(w)=1 b^{b} 1 \cdots \\
& \Delta^{5}(w)=1 b \cdots \\
& \Delta^{6}(w)=1 \cdots \\
& w \text { has the factor }\left(1^{b} b\right)^{\frac{b}{2}}\left(1^{b} b^{b}\right)^{\frac{b}{2}}(1 b)^{\frac{b}{2}} .
\end{aligned}
$$

Case (4) If $p=1 b 1 b 11 b$, then

$$
\begin{aligned}
\Delta^{0}(w)= & \left(\left(1^{b} b\right)^{\frac{b}{2}}\left(\left(1^{b} b^{b}\right)^{\frac{b}{2}} 1\left(b^{b} 1^{b}\right)^{\frac{b}{2}} b\right)^{\frac{b}{4}}\left(\left(1^{b} b^{b}\right)^{\frac{b}{2}}(1 b)^{\frac{b}{2}}\right)^{\frac{b}{2}}\left(1^{b}(b 1)^{\frac{b}{2}} b^{b}(1 b)^{\frac{b}{2}}\right)^{\frac{b}{4}}\right)^{\frac{b}{4}} \\
& \left(\left(1^{b} b\right)^{\frac{b}{2}}\left(1^{b} b^{b}\right)^{\frac{b}{2}}\left(1 b^{b}\right)^{\frac{b}{2}}(1 b)^{\frac{b}{2}}\right)^{\frac{b}{4}}\left(\left(1^{b} b\right)^{\frac{b}{2}}\left(\left(1^{b} b^{b}\right)^{\frac{b}{2}}(1 b)^{\frac{b}{2}}\right)^{\frac{b}{2}}\right)^{\frac{b}{2}} 1^{b}(b 1)^{\frac{b}{2}} b^{b} 1 \cdots \\
\Delta^{1}(w)= & \left((b 1)^{\frac{b}{2}}\left(b^{b} 1\right)^{\frac{b}{2}}\left(b^{b} 1^{b}\right)^{\frac{b}{2}}\left(b 1^{b}\right)^{\frac{b}{2}}\right)^{\frac{b}{4}}\left((b 1)^{\frac{b}{2}} b^{b}(1 b)^{\frac{b}{2}} 1^{b}\right)^{\frac{b}{4}}\left((b 1)^{\frac{b}{2}}\left(b^{b} 1^{b}\right)^{\frac{b}{2}}\right)^{\frac{b}{2}} b 1^{b} b \ldots \\
\Delta^{2}(w)= & \left(1^{b}(b 1)^{\frac{b}{2}} b^{b}(1 b)^{\frac{b}{2}}\right)^{\frac{b}{4}}\left(1^{b} b\right)^{\frac{b}{2}}\left(1^{b} b^{b}\right)^{\frac{b}{2}} 1 b \ldots \\
\Delta^{3}(w)= & \left(b 1^{b}\right)^{\frac{b}{2}}(b 1)^{\frac{b}{2}} b^{b} 1 \ldots \\
\Delta^{4}(w)= & (1 b)^{\frac{b}{2}} 1^{b} b \ldots \\
\Delta^{5}(w)= & 1^{b} b \cdots \\
\Delta^{6}(w)= & b \cdots
\end{aligned}
$$


$w$ has the factor $\left(1^{b} b\right)^{\frac{b}{2}}\left(\left(1^{b} b^{b}\right)^{\frac{b}{2}}(1 b)^{\frac{b}{2}}\right)^{\frac{b}{2}}$.

Case (5) If $p=1 b 1 b 1 b$, then

$$
\begin{aligned}
\Delta^{0}(w) & =\left(\left(1^{b} b\right)^{\frac{b}{2}}\left(1^{b} b^{b}\right)^{\frac{b}{2}}\left(1 b^{b}\right)^{\frac{b}{2}}(1 b)^{\frac{b}{2}}\right)^{\frac{b}{4}}\left(\left(1^{b} b\right)^{\frac{b}{2}}\left(1^{b} b^{b}\right)^{\frac{b}{2}}(1 b)^{\frac{b}{2}}\right)^{\frac{b}{2}} 1^{b}(b 1)^{\frac{b}{2}} b^{b} 1 \cdots \\
\Delta^{1}(w) & =\left((b 1)^{\frac{b}{2}} b^{b}(1 b)^{\frac{b}{2}} 1^{b}\right)^{\frac{b}{4}}\left((b 1)^{\frac{b}{2}}\left(b^{b} 1^{b}\right)^{\frac{b}{2}}\right)^{\frac{b}{2}} b 1^{b} b \cdots \\
\Delta^{2}(w) & =\left(1^{b} b\right)^{\frac{b}{2}}\left(1^{b} b^{b}\right)^{\frac{b}{2}} 1 b \cdots \\
\Delta^{3}(w) & =(b 1)^{\frac{b}{2}} b^{b} 1 \cdots \\
\Delta^{4}(w) & =1^{b} b \cdots \\
\Delta^{5}(w) & =b \cdots
\end{aligned}
$$

$w$ has the factor $\left(1^{b} b\right)^{\frac{b}{2}}\left(1^{b} b^{b}\right)^{\frac{b}{2}}(1 b)^{\frac{b}{2}}$.

Case (6) If $p=1 b 1 b b 111$, then

$$
\begin{aligned}
& \Delta^{0}(w)=\left(\left(1^{b} b\right)^{\frac{b}{2}}\left(\left(1^{b} b^{b}\right)^{\frac{b}{2}}(1 b)^{\frac{b}{2}}\right)^{\frac{b}{2}}\right)^{\frac{b}{2}}\left(\left(1^{b}\left((b 1)^{\frac{b}{2}}\left(b^{b} 1^{b}\right)^{\frac{b}{2}}\right)^{\frac{b}{2}} b\left(\left(1^{b} b^{b}\right)^{\frac{b}{2}}(1 b)^{\frac{b}{2}}\right)^{\frac{b}{2}}\right)^{\frac{b}{4}}\right. \\
&\left.\left(\left(1^{b}(b 1)^{\frac{b}{2}} b^{b}(1 b)^{\frac{b}{2}}\right)^{\frac{b}{4}}\left(\left(1^{b} b\right)^{\frac{b}{2}}\left(\left(1^{b} b^{b}\right)^{\frac{b}{2}}(1 b)^{\frac{b}{2}}\right)^{\frac{b}{2}}\right)^{\frac{b}{2}}\right)^{\frac{b}{2}}\right)^{\frac{b}{2}} 1^{b}\left(\left((b 1)^{\frac{b}{2}}\left(b^{b} 1^{b}\right)^{\frac{b}{2}}\right)^{\frac{b}{2}}\left(b 1^{b}\right)^{\frac{b}{2}}\right)^{\frac{b}{2}}(b 1)^{\frac{b}{2}} b^{b} 1 \cdots \\
& \Delta^{1}(w)=\left((b 1)^{\frac{b}{2}}\left(b^{b} 1^{b}\right)^{\frac{b}{2}}\right)^{\frac{b}{2}}\left(\left(b\left(1^{b} b^{b}\right)^{\frac{b}{2}} 1\left(b^{b} 1^{b}\right)^{\frac{b}{2}}\right)^{\frac{b}{4}}\left(\left(b 1^{b}\right)^{\frac{b}{2}}\left((b 1)^{\frac{b}{2}}\left(b^{b} 1^{b}\right)^{\frac{b}{2}}\right)^{\frac{b}{2}}\right)^{\frac{b}{2}}\right)^{\frac{b}{2}} b\left(\left(1^{b} b^{b}\right)^{\frac{b}{2}}(1 b)^{\frac{b}{2}}\right)^{\frac{b}{2}} 1^{b} b \cdots \\
& \Delta^{2}(w)=\left(1^{b} b^{b}\right)^{\frac{b}{2}}\left(\left(1 b^{b}\right)^{\frac{b}{2}}\left((1 b)^{\frac{b}{2}}\left(1^{b} b^{b}\right)^{\frac{b}{2}}\right)^{\frac{b}{2}}\right)^{\frac{b}{2}} 1\left(b^{b} 1^{b}\right)^{\frac{b}{2}} b 1 \cdots \\
& \Delta^{3}(w)= b^{b}\left((1 b)^{\frac{b}{2}}\left(1^{b} b^{b}\right)^{\frac{b}{2}}\right)^{\frac{b}{2}} 1 b^{b} 1 \ldots \\
& \Delta^{4}(w)= b\left(1^{b} b^{b}\right)^{\frac{b}{2}} 1 b \cdots \\
& \Delta^{5}(w)= 1 b^{b} 1 \ldots \\
& \Delta^{6}(w)= 1 b \cdots \\
& \Delta^{7}(w)= 1 \cdots \\
& w \text { has the factor } 1^{b}\left(b 1^{b}\right)^{\frac{b}{2}}(b 1)^{\frac{b}{2}} \text { in }\left(\left((b 1)^{\frac{b}{2}}\left(b^{b} 1^{b}\right)^{\frac{b}{2}}\right)^{\frac{b}{2}}\left(b 1^{b}\right)^{\frac{b}{2}}\right)^{\frac{b}{2}} .
\end{aligned}
$$

Case (7) If $p=1 b 1 b b 11 b$, then

$$
\begin{aligned}
\Delta^{0}(w)= & \left(\left(\left(1^{b} b\right)^{\frac{b}{2}}\left(\left(1^{b} b^{b}\right)^{\frac{b}{2}}(1 b)^{\frac{b}{2}}\right)^{\frac{b}{2}}\right)^{\frac{b}{2}}\left(1^{b}\left((b 1)^{\frac{b}{2}}\left(b^{b} 1^{b}\right)^{\frac{b}{2}}\right)^{\frac{b}{2}} b\left(\left(1^{b} b^{b}\right)^{\frac{b}{2}}(1 b)^{\frac{b}{2}}\right)^{\frac{b}{2}}\right)^{\frac{b}{4}}\right. \\
& \left.\left(1^{b}(b 1)^{\frac{b}{2}} b^{b}(1 b)^{\frac{b}{2}}\right)^{\frac{b}{4}}\left(\left(1^{b} b\right)^{\frac{b}{2}}\left(1^{b} b^{b}\right)^{\frac{b}{2}}\left(1 b^{b}\right)^{\frac{b}{2}}(1 b)^{\frac{b}{2}}\right)^{\frac{b}{2}}\right)^{\frac{b}{4}} \\
& \left(\left(1^{b} b\right)^{\frac{b}{2}}\left(\left(1^{b} b^{b}\right)^{\frac{b}{2}}(1 b)^{\frac{b}{2}}\right)^{\frac{b}{2}}\right)^{\frac{b}{2}} 1^{b}\left(\left((b 1)^{\frac{b}{2}}\left(b^{b} 1^{b}\right)^{\frac{b}{2}}\right)^{\frac{b}{2}}\left(b 1^{b}\right)^{\frac{b}{2}}\right)^{\frac{b}{2}}(b 1)^{\frac{b}{2}} \ldots \\
\Delta^{1}(w)= & \left(\left((b 1)^{\frac{b}{2}}\left(b^{b} 1^{b}\right)^{\frac{b}{2}}\right)^{\frac{b}{2}}\left(b\left(1^{b} b^{b}\right)^{\frac{b}{2}} 1\left(b^{b} 1^{b}\right)^{\frac{b}{2}}\right)^{\frac{b}{4}}\left(b 1^{b}\right)^{\frac{b}{2}}\left((b 1)^{\frac{b}{2}} b^{b}(1 b)^{\frac{b}{2}} 1^{b}\right)^{\frac{b}{4}}\right)^{\frac{b}{4}} \\
& \left(\left((b 1)^{\frac{b}{2}}\left(b^{b} 1^{b}\right)^{\frac{b}{2}}\right)^{\frac{b}{2}} b\left(\left(1^{b} b^{b}\right)^{\frac{b}{2}}(1 b)^{\frac{b}{2}}\right)^{\frac{b}{2}} 1^{b}\right)^{\frac{b}{4}}\left(\left((b 1)^{\frac{b}{2}}\left(b^{b} 1^{b}\right)^{\frac{b}{2}}\right)^{\frac{b}{2}}\left(b 1^{b}\right)^{\frac{b}{2}}\right)^{\frac{b}{2}}(b 1)^{\frac{b}{2}} b^{b} 1 b \ldots \\
\Delta^{2}(w)= & \left(\left(1^{b} b^{b}\right)^{\frac{b}{2}}\left(1 b^{b}\right)^{\frac{b}{2}}(1 b)^{\frac{b}{2}}\left(1^{b} b\right)^{\frac{b}{2}}\right)^{\frac{b}{4}}\left(\left(1^{b} b^{b}\right)^{\frac{b}{2}} 1\left(b^{b} 1^{b}\right)^{\frac{b}{2}} b\right)^{\frac{b}{4}}\left(\left(1^{b} b^{b}\right)^{\frac{b}{2}}(1 b)^{\frac{b}{2}}\right)^{\frac{b}{2}} 1^{b} b 1 \ldots \\
\Delta^{3}(w)= & \left(b^{b}(1 b)^{\frac{b}{2}} 1^{b}(b 1)^{\frac{b}{2}}\right)^{\frac{b}{4}}\left(b^{b} 1\right)^{\frac{b}{2}}\left(b^{b} 1^{b}\right)^{\frac{b}{2}} b 1 \ldots \\
\Delta^{4}(w)= & \left(b 1^{b}\right)^{\frac{b}{2}}(b 1)^{\frac{b}{2}} b^{b} 1 \ldots \\
\Delta^{5}(w)= & (1 b)^{\frac{b}{2}} 1^{b} b \ldots \\
\Delta^{6}(w)= & 1^{b} b \cdots \\
\Delta^{7}(w)= & b \cdots
\end{aligned}
$$

$w$ has the factor $1^{b}\left(b 1^{b}\right)^{\frac{b}{2}}(b 1)^{\frac{b}{2}}$. 
Case (8) If $p=1 b 1 b b 1 b$, then

$$
\begin{aligned}
\Delta^{0}(w) & =\left(\left(\left(1^{b} b\right)^{\frac{b}{2}}\left(\left(1^{b} b^{b}\right)^{\frac{b}{2}}(1 b)^{\frac{b}{2}}\right)^{\frac{b}{2}}\right)^{\frac{b}{2}} 1^{b}\left(\left((b 1)^{\frac{b}{2}}\left(b^{b} 1^{b}\right)^{\frac{b}{2}}\right)^{\frac{b}{2}}\left(b 1^{b}\right)^{\frac{b}{2}}\right)^{\frac{b}{2}}(b 1)^{\frac{b}{2}} \ldots\right. \\
\Delta^{1}(w) & =\left(\left((b 1)^{\frac{b}{2}}\left(b^{b} 1^{b}\right)^{\frac{b}{2}}\right)^{\frac{b}{2}} b\left(\left(1^{b} b^{b}\right)^{\frac{b}{2}}(1 b)^{\frac{b}{2}}\right)^{\frac{b}{2}} 1^{b}\right)^{\frac{b}{4}}\left(\left((b 1)^{\frac{b}{2}}\left(b^{b} 1^{b}\right)^{\frac{b}{2}}\right)^{\frac{b}{2}}\left(b 1^{b}\right)^{\frac{b}{2}}\right)^{\frac{b}{2}}(b 1)^{\frac{b}{2}} b^{b} 1 \ldots \\
\Delta^{2}(w) & =\left(\left(1^{b} b^{b}\right)^{\frac{b}{2}} 1\left(b^{b} 1^{b}\right)^{\frac{b}{2}} b\right)^{\frac{b}{4}}\left(\left(1^{b} b^{b}\right)^{\frac{b}{2}}(1 b)^{\frac{b}{2}}\right)^{\frac{b}{2}} 1^{b} b \cdots \\
\Delta^{3}(w) & =\left(b^{b} 1\right)^{\frac{b}{2}}\left(b^{b} 1^{b}\right)^{\frac{b}{2}} b 1 \cdots \\
\Delta^{4}(w) & =(b 1)^{\frac{b}{2}} b^{b} 1 \ldots \\
\Delta^{5}(w) & =1^{b} b \cdots \\
\Delta^{6}(w) & =b \cdots
\end{aligned}
$$

Case (9) If $p=1 b 1 b b b 111$, then

$$
\begin{aligned}
& \Delta^{0}(w)=\left(\left(\left(1^{b} b\right)^{\frac{b}{2}}\left(\left(1^{b} b^{b}\right)^{\frac{b}{2}}(1 b)^{\frac{b}{2}}\right)^{\frac{b}{2}}\right)^{\frac{b}{2}}\left(1^{b}(b 1)^{\frac{b}{2}} b^{b}(1 b)^{\frac{b}{2}}\right)^{\frac{b}{4}}\right)^{\frac{b}{2}}\left(\left(\left(1^{b} b\right)^{\frac{b}{2}}\left(\left(1^{b} b^{b}\right)^{\frac{b}{2}} 1\left(b^{b} 1^{b}\right)^{\frac{b}{2}} b\right)^{\frac{b}{4}}\right.\right. \\
& \left.\left(\left(1^{b} b^{b}\right)^{\frac{b}{2}}(1 b)^{\frac{b}{2}}\right)^{\frac{b}{2}}\left(1^{b}(b 1)^{\frac{b}{2}} b^{b}(1 b)^{\frac{b}{2}}\right)^{\frac{b}{4}}\right)^{\frac{b}{4}}\left(\left(\left(1^{b} b\right)^{\frac{b}{2}}\left(1^{b} b^{b}\right)^{\frac{b}{2}}\left(1 b^{b}\right)^{\frac{b}{2}}(1 b)^{\frac{b}{2}}\right)^{\frac{b}{4}}\right. \\
& \left.\left.\left(\left(\left(1^{b} b\right)^{\frac{b}{2}}\left(\left(1^{b} b^{b}\right)^{\frac{b}{2}}(1 b)^{\frac{b}{2}}\right)^{\frac{b}{2}}\right)^{\frac{b}{2}}\left(1^{b}(b 1)^{\frac{b}{2}} b^{b}(1 b)^{\frac{b}{2}}\right)^{\frac{b}{4}}\right)^{\frac{b}{2}}\right)^{\frac{b}{2}}\right)^{\frac{b}{2}}\left(1^{b} b\right)^{\frac{b}{2}}\left(\left(\left(1^{b} b^{b}\right)^{\frac{b}{2}} 1\left(b^{b} 1^{b}\right)^{\frac{b}{2}} b\right)^{\frac{b}{4}}\right. \\
& \left.\left(\left(\left(1^{b} b^{b}\right)^{\frac{b}{2}}(1 b)^{\frac{b}{2}}\right)^{\frac{b}{2}}\left(1^{b} b\right)^{\frac{b}{2}}\right)^{\frac{b}{2}}\right)^{\frac{b}{2}}\left(1^{b} b^{b}\right)^{\frac{b}{2}}\left(1 b^{b}\right)^{\frac{b}{2}}(1 b)^{\frac{b}{2}} 1^{b} b \cdots \\
& \Delta^{1}(w)=\left(\left((b 1)^{\frac{b}{2}}\left(b^{b} 1^{b}\right)^{\frac{b}{2}}\right)^{\frac{b}{2}}\left(b 1^{b}\right)^{\frac{b}{2}}\right)^{\frac{b}{2}}\left(( ( b 1 ) ^ { \frac { b } { 2 } } ( b ^ { b } 1 ) ^ { \frac { b } { 2 } } ( b ^ { b } 1 ^ { b } ) ^ { \frac { b } { 2 } } ( b 1 ^ { b } ) ^ { \frac { b } { 2 } } ) ^ { \frac { b } { 4 } } \left(\left((b 1)^{\frac{b}{2}} b^{b}(1 b)^{\frac{b}{2}} 1^{b}\right)^{\frac{b}{4}}\right.\right. \\
& \left.\left.\left(\left((b 1)^{\frac{b}{2}}\left(b^{b} 1^{b}\right)^{\frac{b}{2}}\right)^{\frac{b}{2}}\left(b 1^{b}\right)^{\frac{b}{2}}\right)^{\frac{b}{2}}\right)^{\frac{b}{2}}\right)^{\frac{b}{2}}(b 1)^{\frac{b}{2}}\left(\left(b^{b} 1\right)^{\frac{b}{2}}\left(\left(b^{b} 1^{b}\right)^{\frac{b}{2}}(b 1)^{\frac{b}{2}}\right)^{\frac{b}{2}}\right)^{\frac{b}{2}} b^{b}(1 b)^{\frac{b}{2}} 1^{b} b \ldots \\
& \Delta^{2}(w)=\left(\left(1^{b} b^{b}\right)^{\frac{b}{2}}(1 b)^{\frac{b}{2}}\right)^{\frac{b}{2}}\left(\left(1^{b}(b 1)^{\frac{b}{2}} b^{b}(1 b)^{\frac{b}{2}}\right)^{\frac{b}{4}}\left(\left(1^{b} b\right)^{\frac{b}{2}}\left(\left(1^{b} b^{b}\right)^{\frac{b}{2}}(1 b)^{\frac{b}{2}}\right)^{\frac{b}{2}}\right)^{\frac{b}{2}}\right)^{\frac{b}{2}} 1^{b}\left((b 1)^{\frac{b}{2}}\left(b^{b} 1^{b}\right)^{\frac{b}{2}}\right)^{\frac{b}{2}} b 1^{b} b \ldots \\
& \Delta^{3}(w)=\left(b^{b} 1^{b}\right)^{\frac{b}{2}}\left(\left(b 1^{b}\right)^{\frac{b}{2}}\left((b 1)^{\frac{b}{2}}\left(b^{b} 1^{b}\right)^{\frac{b}{2}}\right)^{\frac{b}{2}}\right)^{\frac{b}{2}} b\left(1^{b} b^{b}\right)^{\frac{b}{2}} 1 b \cdots \\
& \Delta^{4}(w)=b^{b}\left((1 b)^{\frac{b}{2}}\left(1^{b} b^{b}\right)^{\frac{b}{2}}\right)^{\frac{b}{2}} 1 b^{b} 1 \cdots \\
& \Delta^{5}(w)=b\left(1^{b} b^{b}\right)^{\frac{b}{2}} 1 b \cdots \\
& \Delta^{6}(w)=1 b^{b} 1 \cdots \\
& \Delta^{7}(w)=1 b \cdots \\
& \Delta^{8}(w)=1 \cdots
\end{aligned}
$$

Case (10) If $p=1 b 1 b b b 11 b$, then

$$
\begin{aligned}
& \Delta^{0}(w)=\left(\left(\left(1^{b} b\right)^{\frac{b}{2}}\left(\left(1^{b} b^{b}\right)^{\frac{b}{2}}(1 b)^{\frac{b}{2}}\right)^{\frac{b}{2}}\right)^{\frac{b}{2}}\left(1^{b}(b 1)^{\frac{b}{2}} b^{b}(1 b)^{\frac{b}{2}}\right)^{\frac{b}{4}}\right)^{\frac{b}{2}}\left(\left(1^{b} b\right)^{\frac{b}{2}}\left(\left(1^{b} b^{b}\right)^{\frac{b}{2}} 1\left(b^{b} 1^{b}\right)^{\frac{b}{2}} b\right)^{\frac{b}{4}}\right. \\
& \left.\left(\left(1^{b} b^{b}\right)^{\frac{b}{2}}(1 b)^{\frac{b}{2}}\right)^{\frac{b}{2}}\left(1^{b}(b 1)^{\frac{b}{2}} b^{b}(1 b)^{\frac{b}{2}}\right)^{\frac{b}{4}}\right)^{\frac{b}{4}}\left(\left(1^{b} b\right)^{\frac{b}{2}}\left(1^{b} b^{b}\right)^{\frac{b}{2}}\left(1 b^{b}\right)^{\frac{b}{2}}(1 b)^{\frac{b}{2}}\right)^{\frac{b}{4}}\left(\left(\left(1^{b} b\right)^{\frac{b}{2}}\left(\left(1^{b} b^{b}\right)^{\frac{b}{2}}(1 b)^{\frac{b}{2}}\right)^{\frac{b}{2}}\right)^{\frac{b}{2}} 1^{b}\right. \\
& \left(\left((b 1)^{\frac{b}{2}}\left(b^{b} 1^{b}\right)^{\frac{b}{2}}\right)^{\frac{b}{2}}\left(b 1^{b}\right)^{\frac{b}{2}}(b 1)^{\frac{b}{2}} \ldots\right. \\
& \Delta^{1}(w)=\left(\left(\left((b 1)^{\frac{b}{2}}\left(b^{b} 1^{b}\right)^{\frac{b}{2}}\right)^{\frac{b}{2}}\left(b 1^{b}\right)^{\frac{b}{2}}\right)^{\frac{b}{2}}\left((b 1)^{\frac{b}{2}}\left(b^{b} 1\right)^{\frac{b}{2}}\left(b^{b} 1^{b}\right)^{\frac{b}{2}}\left(b 1^{b}\right)^{\frac{b}{2}}\right)^{\frac{b}{4}}\left((b 1)^{\frac{b}{2}} b^{b}(1 b)^{\frac{b}{2}} 1^{b}\right)^{\frac{b}{4}}\right. \\
& \left.\left(\left((b 1)^{\frac{b}{2}}\left(b^{b} 1^{b}\right)^{\frac{b}{2}}\right)^{\frac{b}{2}} b\left(\left(1^{b} b^{b}\right)^{\frac{b}{2}}(1 b)^{\frac{b}{2}}\right)^{\frac{b}{2}} 1^{b}\right)^{\frac{b}{4}}\right)^{\frac{b}{4}}\left(\left(\left(b 1^{b}\right)^{\frac{b}{2}}\left((b 1)^{\frac{b}{2}}\left(b^{b} 1^{b}\right)^{\frac{b}{2}}\right)^{\frac{b}{2}}\right)^{\frac{b}{2}} b\left(\left(\left(1^{b} b^{b}\right)^{\frac{b}{2}}(1 b)^{\frac{b}{2}}\right)^{\frac{b}{2}}\left(1^{b} b\right)^{\frac{b}{2}}\right)^{\frac{b}{2}}\right. \\
& \left.\left(1^{b} b^{b}\right)^{\frac{b}{2}}\right)^{\frac{b}{4}}\left(\left(\left(1 b^{b}\right)^{\frac{b}{2}}\left((1 b)^{\frac{b}{2}}\left(1^{b} b^{b}\right)^{\frac{b}{2}}\right)^{\frac{b}{2}}\right)^{\frac{b}{2}}\left(1\left(b^{b} 1^{b}\right)^{\frac{b}{2}} b\left(1^{b} b^{b}\right)^{\frac{b}{2}}\right)^{\frac{b}{4}}\right)^{\frac{b}{2}}\left(1 b^{b}\right)^{\frac{b}{2}}(1 b)^{\frac{b}{2}} 1^{b} b 1 \ldots \\
& \Delta^{2}(w)=\left(\left(\left(1^{b} b^{b}\right)^{\frac{b}{2}}(1 b)^{\frac{b}{2}}\right)^{\frac{b}{2}}\left(1^{b}(b 1)^{\frac{b}{2}} b^{b}(1 b)^{\frac{b}{2}}\right)^{\frac{b}{4}}\left(1^{b} b\right)^{\frac{b}{2}}\left(\left(1^{b} b^{b}\right)^{\frac{b}{2}} 1\left(b^{b} 1^{b}\right)^{\frac{b}{2}} b\right)^{\frac{b}{4}}\right)^{\frac{b}{4}} \\
& \left(\left((1 b)^{\frac{b}{2}}\left(1^{b} b^{b}\right)^{\frac{b}{2}}\right)^{\frac{b}{2}} 1\left(\left(b^{b} 1^{b}\right)^{\frac{b}{2}}(b 1)^{\frac{b}{2}}\right)^{\frac{b}{2}} b^{b}\right)^{\frac{b}{4}}\left(\left((1 b)^{\frac{b}{2}}\left(1^{b} b^{b}\right)^{\frac{b}{2}}\right)^{\frac{b}{2}}\left(1 b^{b}\right)^{\frac{b}{2}}\right)^{\frac{b}{2}}(1 b)^{\frac{b}{2}} 1^{b} b 1 \ldots \\
& \Delta^{3}(w)=\left(\left(b^{b} 1^{b}\right)^{\frac{b}{2}}\left(b 1^{b}\right)^{\frac{b}{2}}(b 1)^{\frac{b}{2}}\left(b^{b} 1\right)^{\frac{b}{2}}\right)^{\frac{b}{4}}\left(\left(1^{b} b^{b}\right)^{\frac{b}{2}} 1\left(b^{b} 1^{b}\right)^{\frac{b}{2}} b\right)^{\frac{b}{4}}\left(\left(1^{b} b^{b}\right)^{\frac{b}{2}}(1 b)^{\frac{b}{2}}\right)^{\frac{b}{2}} 1^{b} b 1 \cdots \\
& \Delta^{4}(w)=\left(b^{b}(1 b)^{\frac{b}{2}} 1^{b}(b 1)^{\frac{b}{2}}\right)^{\frac{b}{4}}\left(b^{b} 1\right)^{\frac{b}{2}}\left(b^{b} 1^{b}\right)^{\frac{b}{2}} b 1 \cdots
\end{aligned}
$$


Notice that we take $\Delta^{4}(w)$ from Case (7).

$w$ has the factor $\left(\left(\left(1^{b} b\right)^{\frac{b}{2}}\left(\left(1^{b} b^{b}\right)^{\frac{b}{2}}(1 b)^{\frac{b}{2}}\right)^{\frac{b}{2}}\right)^{\frac{b}{2}} 1^{b}\left(\left((b 1)^{\frac{b}{2}}\left(b^{b} 1^{b}\right)^{\frac{b}{2}}\right)^{\frac{b}{2}}\right.\right.$.

Case (11) If $p=1 b 1 b b b 1 b$, there are two subcases to consider.

(i) If $b \neq 4$, there are again two subcases.

(a) If $b / 4$ is odd, then

$$
\begin{aligned}
& \Delta^{0}(w)=\left(\left(\left(1^{b} b\right)^{\frac{b}{2}}\left(\left(1^{b} b^{b}\right)^{\frac{b}{2}}(1 b)^{\frac{b}{2}}\right)^{\frac{b}{2}}\right)^{\frac{b}{2}}\left(1^{b}(b 1)^{\frac{b}{2}} b^{b}(1 b)^{\frac{b}{2}}\right)^{\frac{b}{4}}\right)^{\frac{b}{2}}\left(1^{b} b\right)^{\frac{b}{2}}\left(\left(\left(1^{b} b^{b}\right)^{\frac{b}{2}} 1\left(b^{b} 1^{b}\right)^{\frac{b}{2}} b\right)^{\frac{b}{4}}\right. \\
& \left.\left(\left(\left({ }^{b} b^{b}\right)^{\frac{b}{2}}(1 b)^{\frac{b}{2}}\right)^{\frac{b}{2}}\left(1^{b} b\right)^{\frac{b}{2}}\right)^{\frac{b}{2}}\right)^{\frac{b}{2}}\left(1^{b} b^{b}\right)^{\frac{b}{2}}\left(\left(( ( 1 b ^ { b } ) ^ { \frac { b } { 2 } } ( ( 1 b ) ^ { \frac { b } { 2 } } ( 1 ^ { b } b ^ { b } ) ^ { \frac { b } { 2 } } ) ^ { \frac { b } { 2 } } ) ^ { \frac { b } { 2 } } \left(1\left(b^{b} 1^{b}\right)^{\frac{b}{2}} b\right.\right.\right. \\
& \left.\left.\left(1^{b} b^{b}\right)^{\frac{b}{2}}\right)^{\frac{b}{4}}\right)^{\frac{b}{2}}\left(1 b^{b}\right)^{\frac{b}{2}}\left(\left((1 b)^{\frac{b}{2}} 1^{b}(b 1)^{\frac{b}{2}} b^{b}\right)^{\frac{b}{4}}\left(\left((1 b)^{\frac{b}{2}}\left(1^{b} b^{b}\right)^{\frac{b}{2}}\right)^{\frac{b}{2}}\left(1 b^{b}\right)^{\frac{b}{2}}\right)^{\frac{b}{2}}\right)^{\frac{b}{2}}(1 b)^{\frac{b}{2}} \\
& \left(\left(\left(1^{b} b\right)^{\frac{b}{2}}\left(\left(1^{b} b^{b}\right)^{\frac{b}{2}}(1 b)^{\frac{b}{2}}\right)^{\frac{b}{2}}\right)^{\frac{b}{2}}\left(1^{b}(b 1)^{\frac{b}{2}} b^{b}(1 b)^{\frac{b}{2}}\right)^{\frac{b}{4}}\right)^{\frac{b}{2}}\left(1^{b} b\right)^{\frac{b}{2}}\left(\left(\left(1^{b} b^{b}\right)^{\frac{b}{2}} 1\left(b^{b} 1^{b}\right)^{\frac{b}{2}} b\right)^{\frac{b}{4}}\right. \\
& \left.\left.\left(\left(\left(1^{b} b^{b}\right)^{\frac{b}{2}}(1 b)^{\frac{b}{2}}\right)^{\frac{b}{2}}\left(1^{b} b\right)^{\frac{b}{2}}\right)^{\frac{b}{2}}\right)^{\frac{b}{2}}\left(1^{b} b^{b}\right)^{\frac{b}{2}}\right)^{\frac{b-4}{8}} \ldots \\
& \Delta^{1}(w)=\left(\left((b 1)^{\frac{b}{2}}\left(b^{b} 1^{b}\right)^{\frac{b}{2}}\right)^{\frac{b}{2}}\left(b 1^{b}\right)^{\frac{b}{2}}\right)^{\frac{b}{2}}(b 1)^{\frac{b}{2}}\left(\left(b^{b} 1\right)^{\frac{b}{2}}\left(\left(b^{b} 1^{b}\right)^{\frac{b}{2}}(b 1)^{\frac{b}{2}}\right)^{\frac{b}{2}}\right)^{\frac{b}{2}} b^{b}\left(\left(\left((1 b)^{\frac{b}{2}}\left(1^{b} b^{b}\right)^{\frac{b}{2}}\right)^{\frac{b}{2}}\right.\right. \\
& \left.\left(1 b^{b}\right)^{\frac{b}{2}}\right)^{\frac{b}{2}}(1 b)^{\frac{b}{2}}\left(\left(1^{b} b\right)^{\frac{b}{2}}\left(\left(1^{b} b^{b}\right)^{\frac{b}{2}}(1 b)^{\frac{b}{2}}\right)^{\frac{b}{2}}\right)^{\frac{b}{2}} 1^{b}\left(\left((b 1)^{\frac{b}{2}}\left(b^{b} 1^{b}\right)^{\frac{b}{2}}\right)^{\frac{b}{2}}\left(b 1^{b}\right)^{\frac{b}{2}}\right)^{\frac{b}{2}}(b 1)^{\frac{b}{2}} \\
& \left.\left(\left(b^{b} 1\right)^{\frac{b}{2}}\left(\left(b^{b} 1^{b}\right)^{\frac{b}{2}}(b 1)^{\frac{b}{2}}\right)^{\frac{b}{2}}\right)^{\frac{b}{2}} b^{b}\right)^{\frac{b-4}{8}}\left(\left(\left((1 b)^{\frac{b}{2}}\left(1^{b} b^{b}\right)^{\frac{b}{2}}\right)^{\frac{b}{2}}\left(1 b^{b}\right)^{\frac{b}{2}}\right)^{\frac{b}{2}}\left((1 b)^{\frac{b}{2}} 1^{b}(b 1)^{\frac{b}{2}} b^{b}\right)^{\frac{b}{4}}\right)^{\frac{b}{2}} \\
& \left((1 b)^{\frac{b}{2}}\left(1^{b} b^{b}\right)^{\frac{b}{2}}\right)^{\frac{b}{2}} 1\left(b^{b} 1^{b}\right)^{\frac{b}{2}} b 1 \cdots \\
& \Delta^{2}(w)=\left(\left(\left(1^{b} b^{b}\right)^{\frac{b}{2}}(1 b)^{\frac{b}{2}}\right)^{\frac{b}{2}} 1^{b}\left((b 1)^{\frac{b}{2}}\left(b^{b} 1^{b}\right)^{\frac{b}{2}}\right)^{\frac{b}{2}} b\right)^{\frac{b}{4}}\left(\left(\left(1^{b} b^{b}\right)^{\frac{b}{2}}(1 b)^{\frac{b}{2}}\right)^{\frac{b}{2}}\left(1^{b} b\right)^{\frac{b}{2}}\right)^{\frac{b}{2}}\left(1^{b} b^{b}\right)^{\frac{b}{2}} 1 b^{b} 1 \cdots \\
& \Delta^{3}(w)=\left(\left(b^{b} 1^{b}\right)^{\frac{b}{2}} b\left(1^{b} b^{b}\right)^{\frac{b}{2}} 1\right)^{\frac{b}{4}}\left(\left(b^{b} 1^{b}\right)^{\frac{b}{2}}(b 1)^{\frac{b}{2}}\right)^{\frac{b}{2}} b^{b} 1 b \cdots \\
& \Delta^{4}(w)=\left(b^{b} 1\right)^{\frac{b}{2}}\left(b^{b} 1^{b}\right)^{\frac{b}{2}} b 1 \cdots \\
& \Delta^{5}(w)=(b 1)^{\frac{b}{2}} b^{b} 1 \cdots \\
& \Delta^{6}(w)=1^{b} b \cdots \\
& \Delta^{7}(w)=b \cdots
\end{aligned}
$$

(b) If $b / 4$ is even, then

$$
\begin{aligned}
\Delta^{0}(w) & =\left(( ( ( 1 ^ { b } b ) ^ { \frac { b } { 2 } } ( ( 1 ^ { b } b ^ { b } ) ^ { \frac { b } { 2 } } ( 1 b ) ^ { \frac { b } { 2 } } ) ^ { \frac { b } { 2 } } ) ^ { \frac { b } { 2 } } ( 1 ^ { b } ( b 1 ) ^ { \frac { b } { 2 } } b ^ { b } ( 1 b ) ^ { \frac { b } { 2 } } ) ^ { \frac { b } { 4 } } ) ^ { \frac { b } { 2 } } ( 1 ^ { b } b ) ^ { \frac { b } { 2 } } \left(\left(\left(1^{b} b^{b}\right)^{\frac{b}{2}} 1\left(b^{b} 1^{b}\right)^{\frac{b}{2}} b\right)^{\frac{b}{4}}\right.\right. \\
& \left.\left.\left(\left(1^{b} b^{b}\right)^{\frac{b}{2}}(1 b)^{\frac{b}{2}}\right)^{\frac{b}{2}}\left(1^{b} b\right)^{\frac{b}{2}}\right)^{\frac{b}{2}}\right)^{\frac{b}{2}}\left(1^{b} b^{b}\right)^{\frac{b}{2}}\left(( ( 1 b ^ { b } ) ^ { \frac { b } { 2 } } ( ( 1 b ) ^ { \frac { b } { 2 } } ( 1 ^ { b } b ^ { b } ) ^ { \frac { b } { 2 } } ) ^ { \frac { b } { 2 } } ) ^ { \frac { b } { 2 } } \left(1\left(b^{b} 1^{b}\right)^{\frac{b}{2}}\right.\right. \\
& \left.\left.\left.b\left(1^{b} b^{b}\right)^{\frac{b}{2}}\right)^{\frac{b}{4}}\right)^{\frac{b}{2}}\left(1 b^{b}\right)^{\frac{b}{2}}\left(\left((1 b)^{\frac{b}{2}} 1^{b}(b 1)^{\frac{b}{2}} b^{b}\right)^{\frac{b}{4}}\left(\left((1 b)^{\frac{b}{2}}\left(1^{b} b^{b}\right)^{\frac{b}{2}}\right)^{\frac{b}{2}}\left(1 b^{b}\right)^{\frac{b}{2}}\right)^{\frac{b}{2}}\right)^{\frac{b}{2}}(1 b)^{\frac{b}{2}}\right)^{\frac{b}{8}} \\
& \left(\left(\left(1^{b} b\right)^{\frac{b}{2}}\left(\left(1^{b} b^{b}\right)^{\frac{b}{2}}(1 b)^{\frac{b}{2}}\right)^{\frac{b}{2}}\right)^{\frac{b}{2}}\left(1^{b}(b 1)^{\frac{b}{2}} b^{b}(1 b)^{\frac{b}{2}}\right)^{\frac{b}{4}}\right)^{\frac{b}{2}}\left(\left(1^{b} b\right)^{\frac{b}{2}}\left(1^{b} b^{b}\right)^{\frac{b}{2}}\right. \\
& \left.\left.\left(1 b^{b}\right)^{\frac{b}{2}}(1 b)^{\frac{b}{2}}\right)^{\frac{b}{4}}\right)^{\frac{b}{2}}\left(\left(1^{b} b\right)^{\frac{b}{2}}\left(\left(1^{b} b^{b}\right)^{\frac{b}{2}}(1 b)^{\frac{b}{2}}\right)^{\frac{b}{2}}\right)^{\frac{b}{2}} 1^{b}\left((b 1)^{\frac{b}{2}}\left(b^{b} 1^{b}\right)^{\frac{b}{2}}\right)^{\frac{b}{2}} b 1^{b} b \ldots \\
\Delta^{1}(w) & =\left(\left(\left((b 1)^{\frac{b}{2}}\left(b^{b} 1^{b}\right)^{\frac{b}{2}}\right)^{\frac{b}{2}}\left(b 1^{b}\right)^{\frac{b}{2}}\right)^{\frac{b}{2}}(b 1)^{\frac{b}{2}}\left(\left(b^{b} 1\right)^{\frac{b}{2}}\left(\left(b^{b} 1^{b}\right)^{\frac{b}{2}}(b 1)^{\frac{b}{2}}\right)^{\frac{b}{2}}\right)^{\frac{b}{2}} b^{b}\right. \\
& \left.\left(\left((1 b)^{\frac{b}{2}}\left(1^{b} b^{b}\right)^{\frac{b}{2}}\right)^{\frac{b}{2}}\left(1 b^{b}\right)^{\frac{b}{2}}\right)^{\frac{b}{2}}(1 b)^{\frac{b}{2}}\left(\left(1^{b} b\right)^{\frac{b}{2}}\left(\left(1^{b} b^{b}\right)^{\frac{b}{2}}(1 b)^{\frac{b}{2}}\right)^{\frac{b}{2}}\right)^{\frac{b}{2}} 1^{b}\right)^{\frac{b}{8}} \\
& \left(\left(\left((b 1)^{\frac{b}{2}}\left(b^{b} 1^{b}\right)^{\frac{b}{2}}\right)^{\frac{b}{2}}\left(b 1^{b}\right)^{\frac{b}{2}}\right)^{\frac{b}{2}}\left((b 1)^{\frac{b}{2}} b^{b}(1 b)^{\frac{b}{2}} 1^{b}\right)^{\frac{b}{4}}\right)^{\frac{b}{2}}\left((b 1)^{\frac{b}{2}}\left(b^{b} 1^{b}\right)^{\frac{b}{2}}\right)^{\frac{b}{2}} b\left(1^{b} b^{b}\right)^{\frac{b}{2}} 1 b \ldots
\end{aligned}
$$

Notice that the $\Delta^{i}(w)$ 's are the same as in $(a)$ for $2 \leq i \leq 7$.

$$
w \text { has the factor }\left(\left(1^{b} b\right)^{\frac{b}{2}}\left(\left(1^{b} b^{b}\right)^{\frac{b}{2}}(1 b)^{\frac{b}{2}}\right)^{\frac{b}{2}}\right)^{\frac{b}{2}} 1^{b}\left((b 1)^{\frac{b}{2}}\left(b^{b} 1^{b}\right)^{\frac{b}{2}}\right)^{\frac{b}{2}} .
$$

(ii) If $b=4$, there are again 3 subcases to consider. 
(a) If $p=1414441411$, then

$\Delta^{0}(w)=\left(\left(\left(1^{4} 4\right)^{2}\left(\left(1^{4} 4^{4}\right)^{2}(14)^{2}\right)^{2}\right)^{2} 1^{4}(41)^{2} 4^{4}(14)^{2}\right)^{2}\left(1^{4} 4\right)^{2}\left(\left(1^{4} 4^{4}\right)^{2} 1\left(4^{4} 1^{4}\right)^{2} 4\left(\left(\left(1^{4} 4^{4}\right)^{2}(14)^{2}\right)^{2}\right.\right.$

$\left.\left.\left(1^{4} 4\right)^{2}\right)^{2}\right)^{2}\left(1^{4} 4^{4}\right)^{2}\left(\left(\left(\left(14^{4}\right)^{2}\left((14)^{2}\left(1^{4} 4^{4}\right)^{2}\right)^{2}\right)^{2} 1\left(4^{4} 1^{4}\right)^{2} 4\left(1^{4} 4^{4}\right)^{2}\right)^{2}\left(14^{4}\right)^{2}(14)^{2}\left(1^{4} 4\right)^{2}\right.$

$\left.\left(1^{4} 4^{4}\right)^{2}\right)^{2}\left(\left(14^{4}\right)^{2}\left((14)^{2}\left(1^{4} 4^{4}\right)^{2}\right)^{2}\right)^{2}\left(1\left(\left(4^{4} 1^{4}\right)^{2}(41)^{2}\right)^{2} 4^{4}\left((14)^{2}\left(1^{4} 4^{4}\right)^{2}\right)^{2}\left(1\left(4^{4} 1^{4}\right)^{2} 4\left(1^{4} 4^{4}\right)^{2}\right.\right.$

$\left.\left.\left(\left(14^{4}\right)^{2}\left((14)^{2}\left(1^{4} 4^{4}\right)^{2}\right)^{2}\right)^{2}\right)^{2}\right)^{2} 1\left(\left(\left(\left(\left(4^{4} 1^{4}\right)^{2}(41)^{2}\right)^{2}\left(4^{4} 1\right)^{2}\right)^{2}\left(4^{4} 1^{4}\right)^{2} 4\left(1^{4} 4^{4}\right)^{2} 1\right)^{2}\right.$

$\left.\left(\left(4^{4} 1^{4}\right)^{2}(41)^{2}\right)^{2} 4^{4}\left((14)^{2}\left(1^{4} 4^{4}\right)^{2}\right)^{2} 1\right)^{2}\left(\left(\left(4^{4} 1^{4}\right)^{2}(41)^{2}\right)^{2}\left(4^{4} 1\right)^{2}\right)^{2}\left(4^{4} \underline{1}^{4}\right)^{2}$

$\left(\left(41^{4}\right)^{2}\left((41)^{2}\left(4^{4} 1^{4}\right)^{2}\right)^{2}\right)^{2} 4 \cdots$

$\Delta^{1}(w)=\left(\left((41)^{2}\left(4^{4} 1^{4}\right)^{2}\right)^{2}\left(41^{4}\right)^{2}\right)^{2}(41)^{2}\left(\left(4^{4} 1\right)^{2}\left(\left(4^{4} 1^{4}\right)^{2}(41)^{2}\right)^{2}\right)^{2} 4^{4}\left(\left(\left((14)^{2}\left(1^{4} 4^{4}\right)^{2}\right)^{2}\left(14^{4}\right)^{2}\right)^{2}(14)^{2}\right.$

$\left.1^{4}(41)^{2} 4^{4}\right)^{2}\left((14)^{2}\left(1^{4} 4^{4}\right)^{2}\right)^{2}\left(1\left(4^{4} 1^{4}\right)^{2} 4\left(1^{4} 4^{4}\right)^{2}\left(\left(14^{4}\right)^{2}\left((14)^{2}\left(1^{4} 4^{4}\right)^{2}\right)^{2}\right)^{2}\right)^{2} 1\left(\left(\left(\left(\left(4^{4} 1^{4}\right)^{2}\right.\right.\right.\right.$

$\left.\left.\left.\left.(41)^{2}\right)^{2}\left(4^{4} 1\right)^{2}\right)^{2}\left(4^{4} 1^{4}\right)^{2} 4\left(1^{4} 4^{4}\right)^{2} 1\right)^{2}\left(\left(4^{4} 1^{4}\right)^{2}(41)^{2}\right)^{2} 4^{4}\left((14)^{2}\left(1^{4} 4^{4}\right)^{2}\right)^{2} 1\right)^{2} \ldots$

$\Delta^{2}(w)=\left(\left(1^{4} 4^{4}\right)^{2}(14)^{2}\right)^{2} 1^{4}\left((41)^{2}\left(4^{4} 1^{4}\right)^{2}\right)^{2} 4\left(\left(\left(1^{4} 4^{4}\right)^{2}(14)^{2}\right)^{2}\left(1^{4} 4\right)^{2}\right)^{2}\left(1^{4} 4^{4}\right)^{2}\left(\left(14^{4}\right)^{2}\left((14)^{2}\right.\right.$

$\left.\left.\left(1^{4} 4^{4}\right)^{2}\right)^{2}\right)^{2} 1\left(\left(\left(\left(4^{4} 1^{4}\right)^{2}(41)^{2}\right)^{2}\left(4^{4} 1\right)^{2}\right)^{2}\left(4^{4} 1^{4}\right)^{2} 4\left(1^{4} 4\right)^{2} 1\right)^{2} \ldots$

$\Delta^{3}(w)=\left(4^{4} 1^{4}\right)^{2} 4\left(1^{4} 4^{4}\right)^{2} 1\left(\left(4^{4} 1^{4}\right)^{2}(41)^{2}\right)^{2} 4^{4}\left((14)^{2}\left(1^{4} 4^{4}\right)^{2}\right)^{2} 1\left(\left(\left(4^{4} 1^{4}\right)^{2}(41)^{2}\right)^{2}\left(4^{4} 1\right)^{2}\right)^{2} \ldots$

$\Delta^{4}(w)=\left(4^{4} 1\right)^{2}\left(4^{4} 1^{4}\right)^{2} 4\left(1^{4} 4^{4}\right)^{2} 1\left(\left(4^{4} 1^{4}\right)^{2}(41)^{2}\right)^{2} \cdots$

$\Delta^{5}(w)=(41)^{2}\left(4^{4} 1\right)^{2}\left(4^{4} 1^{4}\right)^{2} \cdots$

$\Delta^{6}(w)=1^{4}(41)^{2} 4^{4} \cdots$

$\Delta^{7}(w)=41^{4} 4 \cdots$

$\Delta^{8}(w)=14 \cdots$

$\Delta^{9}(w)=1 \cdots$

$w$ has the factor $1^{4}\left(41^{4}\right)^{2}(41)^{2}$.

(b) If $p=1414441414$, then

$\Delta^{0}(w)=\left(\left(\left(1^{4} 4\right)^{2}\left(\left(1^{4} 4^{4}\right)^{2}(14)^{2}\right)^{2}\right)^{2} 1^{4}(41)^{2} 4^{4}(14)^{2}\right)^{2}\left(1^{4} 4\right)^{2}\left(\left(1^{4} 4^{4}\right)^{2} 1\left(4^{4} 1^{4}\right)^{2} 4\right.$

$\left.\left(\left(\left(1^{4} 4^{4}\right)^{2}(14)^{2}\right)^{2}\left(1^{4} 4\right)^{2}\right)^{2}\right)^{2}\left(1^{4} 4^{4}\right)^{2}\left(\left(\left(\left(14^{4}\right)^{2}\left((14)^{2}\left(1^{4} 4^{4}\right)^{2}\right)^{2}\right)^{2} 1\left(4^{4} 1^{4}\right)^{2} 4\left(1^{4} 4^{4}\right)^{2}\right)^{2}\right.$

$\left.\left(14^{4}\right)^{2}(14)^{2}\left(1^{4} 4\right)^{2}\left(1^{4} 4^{4}\right)^{2}\right)^{2}\left(\left(14^{4}\right)^{2}\left((14)^{2}\left(1^{4} 4^{4}\right)^{2}\right)^{2}\right)^{2} 1\left(\left(4^{4} 1^{4}\right)^{2}(41)^{2}\right)^{2} 4^{4}\left((14)^{2}\right.$

$\left.\left(1^{4} 4^{4}\right)^{2}\right)^{2} 1\left(4^{4} 1^{4}\right)^{2} 4\left(1^{4} 4^{4}\right)^{2}\left(14^{4}\right)^{2}(14)^{2}\left(1^{4} 4\right)^{2}\left(1^{4} 4^{4}\right)^{2}\left(\left(14^{4}\right)^{2}\left((14)^{2}\left(1^{4} 4^{4}\right)^{2}\right)^{2}\right)^{2} 1$

$\left(\left(\left(4^{4} 1^{4}\right)^{2}(41)^{2}\right)^{2}\left(4^{4} 1\right)^{2}\right)^{2}\left(4^{4} \underline{1}^{4}\right)^{2}\left(41^{4}\right)^{2}(41)^{2} 4^{4} 1 \cdots$

$\Delta^{1}(w)=\left(\left((41)^{2}\left(4^{4} 1^{4}\right)^{2}\right)^{2}\left(41^{4}\right)^{2}\right)^{2}(41)^{2}\left(\left(4^{4} 1\right)^{2}\left(\left(4^{4} 1^{4}\right)^{2}(41)^{2}\right)^{2}\right)^{2} 4^{4}\left(\left(\left((14)^{2}\left(1^{4} 4^{4}\right)^{2}\right)^{2}\left(14^{4}\right)^{2}\right)^{2}\right.$

$\left.(14)^{2} 1^{4}(41)^{2} 4^{4}\right)^{2}\left((14)^{2}\left(1^{4} 4^{4}\right)^{2}\right)^{2} 1\left(4^{4} 1^{4}\right)^{2} 4\left(1^{4} 4^{4}\right)^{2}\left(14^{4}\right)^{2}(14)^{2} 1^{4}(41)^{2} 4^{4}$

$\left((14)^{2}\left(1^{4} 4^{4}\right)^{2}\right)^{2} 1\left(\left(4^{4} 1^{4}\right)^{2}(41)^{2}\right)^{2} 4^{4}(14)^{2} 1^{4} 4 \cdots$

$\Delta^{2}(w)=\left(\left(1^{4} 4^{4}\right)^{2}(14)^{2}\right)^{2} 1^{4}\left((41)^{2}\left(4^{4} 1^{4}\right)^{2}\right)^{2} 4\left(\left(\left(1^{4} 4^{4}\right)^{2}(14)^{2}\right)^{2}\left(1^{4} 4\right)^{2}\right)^{2}\left(1^{4} 4^{4}\right)^{2}\left(14^{4}\right)^{2}$

$(14)^{2}\left(1^{4} 4\right)^{2}\left(1^{4} 4^{4}\right)^{2} 1\left(4^{4} 1^{4}\right)^{2} 41^{4} 4 \cdots$

$\Delta^{3}(w)=\left(4^{4} 1^{4}\right)^{2} 4\left(1^{4} 4^{4}\right)^{2} 1\left(\left(4^{4} 1^{4}\right)^{2}(41)^{2}\right)^{2} 4^{4}(14)^{2} 1^{4}(41)^{2}\left(4^{4} 1\right)^{2} 4 \cdots$

$\Delta^{4}(w)=\left(4^{4} 1\right)^{2}\left(4^{4} 1^{4}\right)^{2}\left(41^{4}\right)^{2}(41)^{2} \ldots$

$\Delta^{5}(w)=(41)^{2} 4^{4}(14)^{2} 1^{4} \ldots$

$\Delta^{6}(w)=\left(1^{4} 4\right)^{2} \ldots$

$\Delta^{7}(w)=(41)^{2} \ldots$

$\Delta^{8}(w)=1^{4} \ldots$

$\Delta^{9}(w)=4 \cdots$

$w$ has the factor $1^{4}\left(41^{4}\right)^{2}(41)^{2}$. 
(c) If $p=141444144$, then

$$
\begin{aligned}
\Delta^{0}(w)= & \left(\left(\left(1^{4} 4\right)^{2}\left(\left(1^{4} 4^{4}\right)^{2}(14)^{2}\right)^{2}\right)^{2} 1^{4}(41)^{2} 4^{4}(14)^{2}\right)^{2}\left(1^{4} 4\right)^{2}\left(( 1 ^ { 4 } 4 ^ { 4 } ) ^ { 2 } 1 ( 4 ^ { 4 } 1 ^ { 4 } ) ^ { 2 } 4 \left(\left(\left(1^{4} 4^{4}\right)^{2}(14)^{2}\right)^{2}\right.\right. \\
& \left.\left.\left(1^{4} 4\right)^{2}\right)^{2}\right)^{2}\left(1^{4} 4^{4}\right)^{2}\left(\left(\left(\left(14^{4}\right)^{2}\left((14)^{2}\left(1^{4} 4^{4}\right)^{2}\right)^{2}\right)^{2} 1\left(4^{4} 1^{4}\right)^{2} 4\left(1^{4} 4^{4}\right)\right)^{2}\left(14^{4}\right)^{2}(14)^{2}\right. \\
& \left.\left(1^{4} 4\right)^{2}\left(1^{4} 4^{4}\right)^{2}\right)^{2}\left(\left(14^{4}\right)^{2}\left((14)^{2}\left(1^{4} 4^{4}\right)^{2}\right)^{2}\right)^{2} 1\left(\left(\left(4^{4} 1^{4}\right)^{2}(41)^{2}\right)^{2}\left(4^{4} 1\right)^{2}\right)^{2}\left(4^{4} 1^{4}\right)^{2} \\
& \left(\left(\left(\left(41^{4}\right)^{2}\left((41)^{2}\left(4^{4} 1^{4}\right)^{2}\right)^{2}\right)^{2} 4\left(1^{4} 4^{4}\right)^{2} 1\left(4^{4} 1^{4}\right)\right)^{2}\left(41^{4}\right)^{2}(41)^{2}\left(4^{4} 1\right)^{2}\left(4^{4} 1^{4}\right)^{2}\right)^{2} \ldots \\
\Delta^{1}(w)= & \left(\left((41)^{2}\left(4^{4} 1^{4}\right)^{2}\right)^{2}\left(41^{4}\right)^{2}\right)^{2}(41)^{2}\left(\left(4^{4} 1\right)^{2}\left(\left(4^{4} 1^{4}\right)^{2}(41)^{2}\right)^{2}\right)^{2} 4^{4}\left(\left(\left(\left((14)^{2}\left(1^{4} 4^{4}\right)^{2}\right)^{2}\right.\right.\right. \\
& \left.\left.\left.\left(14^{4}\right)^{2}\right)^{2}(14)^{2} 1^{4}(41)^{2} 4^{4}\right)^{2}\left((14)^{2}\left(1^{4} 4^{4}\right)^{2}\right)^{2} 1\left(\left(4^{4} 1^{4}\right)^{2}(41)^{2}\right)^{2} 4^{4}\right)^{2} \ldots \\
\Delta^{2}(w)= & \left(\left(\left(1^{4} 4^{4}\right)^{2}(14)^{2}\right)^{2} 1^{4}\left((41)^{2}\left(4^{4} 1^{4}\right)^{2}\right)^{2} 4\left(\left(\left(\left(1^{4} 4^{4}\right)^{2}(14)^{2}\right)^{2}\left(1^{4} 4\right)^{2}\right)^{2}\left(1^{4} 4^{4}\right)^{2} 1\left(4^{4} 1^{4}\right)^{2} 4\right)^{2}\right)^{2} \ldots \\
\Delta^{3}(w)= & \left(\left(4^{4} 1^{4}\right)^{2} 4\left(1^{4} 4^{4}\right)^{2} 1\left(\left(\left(4^{4} 1^{4}\right)^{2}(41)^{2}\right)^{2}\left(4^{4} 1\right)^{2}\right)^{2}\right)^{2} \ldots \\
\Delta^{4}(w)= & \left(\left(4^{4} 1\right)^{2}\left(\left(4^{4} 1^{4}\right)^{2}(41)^{2}\right)^{2}\right)^{2} \ldots \\
\Delta^{5}(w)= & \left((41)^{2}\left(4^{4} 1^{4}\right)^{2}\right)^{2} \ldots \\
\Delta^{6}(w)= & \left(1^{4} 4^{4}\right)^{2} \ldots \\
\Delta^{7}(w)= & 4^{4} \cdots \\
\Delta^{8}(w)= & 4 \cdots \\
w \text { has the factor } & 1^{4}\left(41^{4}\right)^{2}(41)^{2} .
\end{aligned}
$$

Case (12) If $p=1 b 1 b b b b$, then

$$
\begin{aligned}
\Delta^{0}(w)= & \left.\left(\left(\left(1^{b} b\right)^{\frac{b}{2}}\left(\left(1^{b} b^{b}\right)^{\frac{b}{2}}(1 b)^{\frac{b}{2}}\right)^{\frac{b}{2}}\right)^{\frac{b}{2}}\left(1^{b}(b 1)^{\frac{b}{2}} b^{b}(1 b)^{\frac{b}{2}}\right)^{\frac{b}{4}}\right)^{\frac{b}{2}}\left(\left(1^{b} b\right)^{\frac{b}{2}}\left(1^{b} b^{b}\right)^{\frac{b}{2}}\left(1 b^{b}\right)^{\frac{b}{2}}(1 b)^{\frac{b}{2}}\right)^{\frac{b}{4}}\right)^{\frac{b}{2}} \\
& \left(\left(1^{b} b\right)^{\frac{b}{2}}\left(\left(1^{b} b^{b}\right)^{\frac{b}{2}}(1 b)^{\frac{b}{2}}\right)^{\frac{b}{2}}\right)^{\frac{b}{2}} 1^{b}\left((b 1)^{\frac{b}{2}}\left(b^{b} 1^{b}\right)^{\frac{b}{2}}\right)^{\frac{b}{2}} b 1^{b} b \cdots \\
\Delta^{1}(w)= & \left(\left(\left((b 1)^{\frac{b}{2}}\left(b^{b} 1^{b}\right)^{\frac{b}{2}}\right)^{\frac{b}{2}}\left(b 1^{b}\right)^{\frac{b}{2}}\right)^{\frac{b}{2}}\left((b 1)^{\frac{b}{2}} b^{b}(1 b)^{\frac{b}{2}} 1^{b}\right)^{\frac{b}{4}}\right)^{\frac{b}{2}}\left((b 1)^{\frac{b}{2}}\left(b^{b} 1^{b}\right)^{\frac{b}{2}}\right)^{\frac{b}{2}} b\left(1^{b} b^{b}\right)^{\frac{b}{2}} 1 b \cdots \\
\Delta^{2}(w)= & \left(\left(\left(1^{b} b^{b}\right)^{\frac{b}{2}}(1 b)^{\frac{b}{2}}\right)^{\frac{b}{2}}\left(1^{b} b\right)^{\frac{b}{2}}\right)^{\frac{b}{2}}\left(1^{b} b^{b}\right)^{\frac{b}{2}} 1 b^{b} 1 \ldots \\
\Delta^{3}(w)= & \left(\left(b^{b} 1^{b}\right)^{\frac{b}{2}}(b 1)^{\frac{b}{2}}\right)^{\frac{b}{2}} b^{b} 1 b \cdots \\
\Delta^{4}(w)= & \left(b^{b} 1^{b}\right)^{\frac{b}{2}} b 1 \ldots \\
\Delta^{5}(w)= & b^{b} 1 \ldots \\
\Delta^{6}(w)= & b \cdots
\end{aligned}
$$

$w$ has the factor $\left(\left(1^{b} b\right)^{\frac{b}{2}}\left(\left(1^{b} b^{b}\right)^{\frac{b}{2}}(1 b)^{\frac{b}{2}}\right)^{\frac{b}{2}}\right)^{\frac{b}{2}} 1^{b}(b 1)^{\frac{b}{2}}\left(b^{b} 1^{b}\right)^{\frac{b}{2}}$.

Case (13) If $p=1 b b 111$, then from Case (6), we have

$w$ has the factor $\left(1^{b} b^{b}\right)^{\frac{b}{2}} 1\left(b^{b} 1^{b}\right)^{\frac{b}{2}}$.

$$
\Delta^{0}(w)=\left(1^{b} b^{b}\right)\left(\left(1 b^{b}\right)^{\frac{b}{2}}\left((1 b)^{\frac{b}{2}}\left(\underline{1}^{b} b^{b}\right)^{\frac{b}{2}}\right)^{\frac{b}{2}}\right)^{\frac{b}{2}} 1\left(b^{b} 1^{b}\right)^{\frac{b}{2}} b 1 \cdots
$$

Case (14) If $p=1 b b 11 b$, using Case (7), we get

$$
\Delta^{0}(w)=\left(\left(1^{b} b^{b}\right)^{\frac{b}{2}}\left(1 b^{b}\right)^{\frac{b}{2}}(1 b)^{\frac{b}{2}}\left(1^{b} b\right)^{\frac{b}{2}}\right)^{\frac{b}{4}}\left(\left(1^{b} b^{b}\right)^{\frac{b}{2}} 1\left(b^{b} 1^{b}\right)^{\frac{b}{2}} b\right)^{\frac{b}{4}}\left(\left(1^{b} b^{b}\right)^{\frac{b}{2}}(1 b)^{\frac{b}{2}}\right)^{\frac{b}{2}} 1^{b} b 1 \cdots
$$

$w$ has the factor $\left(1^{b} b\right)^{\frac{b}{2}}$.

Case (15) If $p=1 b b 1 b$, using Case (8), we get

$w$ has the factor $1^{b} b 1^{b}$.

$$
\Delta^{0}(w)=\left(\left(1^{b} b^{b}\right)^{\frac{b}{2}} 1\left(b^{b} \underline{1}^{b}\right)^{\frac{b}{2}} b\right)^{\frac{b}{4}}\left(\left(1^{b} b^{b}\right)^{\frac{b}{2}}(1 b)^{\frac{b}{2}}\right)^{\frac{b}{2}} 1^{b} b \ldots
$$

Case (16) If $p=1 b b b$, then

$$
\begin{aligned}
\Delta^{0}(w) & =\left(\left(1^{b} b^{b}\right)^{\frac{b}{2}}(1 b)^{\frac{b}{2}}\right)^{\frac{b}{2}} \underline{1}^{b} b 1 \cdots \\
\Delta^{1}(w) & =\left(b^{b} 1^{b}\right)^{\frac{b}{2}} b 1 \cdots \\
\Delta^{2}(w) & =b^{b} 1 \cdots \\
\Delta^{3}(w) & =b \cdots
\end{aligned}
$$

$w$ has the factor $1^{b} b 1$ 


\section{B Details of the proof of Theorem 33}

Case (17) If $p=1 b 11 b 1$, then

$$
\begin{aligned}
\Delta^{0}(w) & =1^{b}(b 1)^{2 n+1}\left(b^{b}(1 b)^{2 n+1} 1^{b}(b 1)^{2 n+1}\right)^{n}\left(b^{b} 1\right)^{2 n+1}\left(b^{b} \underline{1}^{b}\right)^{2 n+1} b 1^{b} b \cdots \\
\Delta^{1}(w) & =\left(b 1^{b}\right)^{2 n+1}(b 1)^{2 n+1} b^{b} 1 b \cdots \\
\Delta^{2}(w) & =(1 b)^{2 n+1} 1^{b} b 1 \cdots \\
\Delta^{3}(w) & =1^{b} b 1 \cdots \\
\Delta^{4}(w) & =b 1 \cdots \\
\Delta^{5}(w) & =1 \cdots
\end{aligned}
$$

$w$ has the factor $1^{b} b 1^{b}$.

Case (18) If $p=1 b 11 b b$, then

$$
\begin{aligned}
\Delta^{0}(w)= & \left(1^{b}(b 1)^{2 n+1}\left(b^{b}(1 b)^{2 n+1} 1^{b}(b 1)^{2 n+1}\right)^{n}\left(\left(b^{b} 1\right)^{2 n+1}\left(\left(b^{b} \underline{1}^{b}\right)^{2 n+1}(b 1)^{2 n+1}\right)^{2 n+1}\right)^{2 n+1}\right)^{2 n+1} \\
& b^{b}(1 b)^{2 n+1}\left(1^{b} b^{b}\right)^{2 n+1} \cdots \\
\Delta^{1}(w)= & \left(\left(b 1^{b}\right)^{2 n+1}\left((b 1)^{2 n+1}\left(b^{b} 1^{b}\right)^{2 n+1}\right)^{2 n+1}\right)^{2 n+1} b\left(1^{b} b^{b}\right)^{2 n+1} 1 b \cdots \\
\Delta^{2}(w)= & \left((1 b)^{2 n+1}\left(1^{b} b^{b}\right)^{2 n+1}\right)^{2 n+1} 1 b^{b} 1 \cdots \\
\Delta^{3}(w)= & \left(1^{b} b^{b}\right)^{2 n+1} 1 b \cdots \\
\Delta^{4}(w)= & b^{b} 1 \cdots \\
\Delta^{5}(w)= & b \cdots
\end{aligned}
$$

$w$ has the factor $1^{b} b 1 b^{b}(1 b) 1^{b}$ if $n=0$ and $1^{b}(b 1)^{2 n+1}\left(b^{b} 1^{b}\right)^{2 n+1}$ in $\left(\left(b^{b} 1^{b}\right)^{2 n+1}(b 1)^{2 n+1}\right)^{2 n+1}$ if $n \neq 0$.

Case (19) If $p=1 b 1 b 11 b 11$, there are two subcases to consider.

(i) If $b=2$, then

$$
\begin{aligned}
& \Delta^{0}(w)=1121122122112122121122122121121122122112122122 \underline{1} 12112122112112212 \ldots \\
& \Delta^{1}(w)=21221221121122121121221221121221211221221 \ldots \\
& \Delta^{2}(w)=112122122112112122112112212 \ldots \\
& \Delta^{3}(w)=21121221211221221 \cdots \\
& \Delta^{4}(w)=12112112212 \cdots \\
& \Delta^{5}(w)=1121221 \cdots \\
& \Delta^{6}(w)=2112 \cdots \\
& \Delta^{7}(w)=12 \cdots \\
& \Delta^{8}(w)=1 \cdots
\end{aligned}
$$

$w$ has the factor 1121121 
(ii) If $b \neq 2$, then $n \neq 0$ and

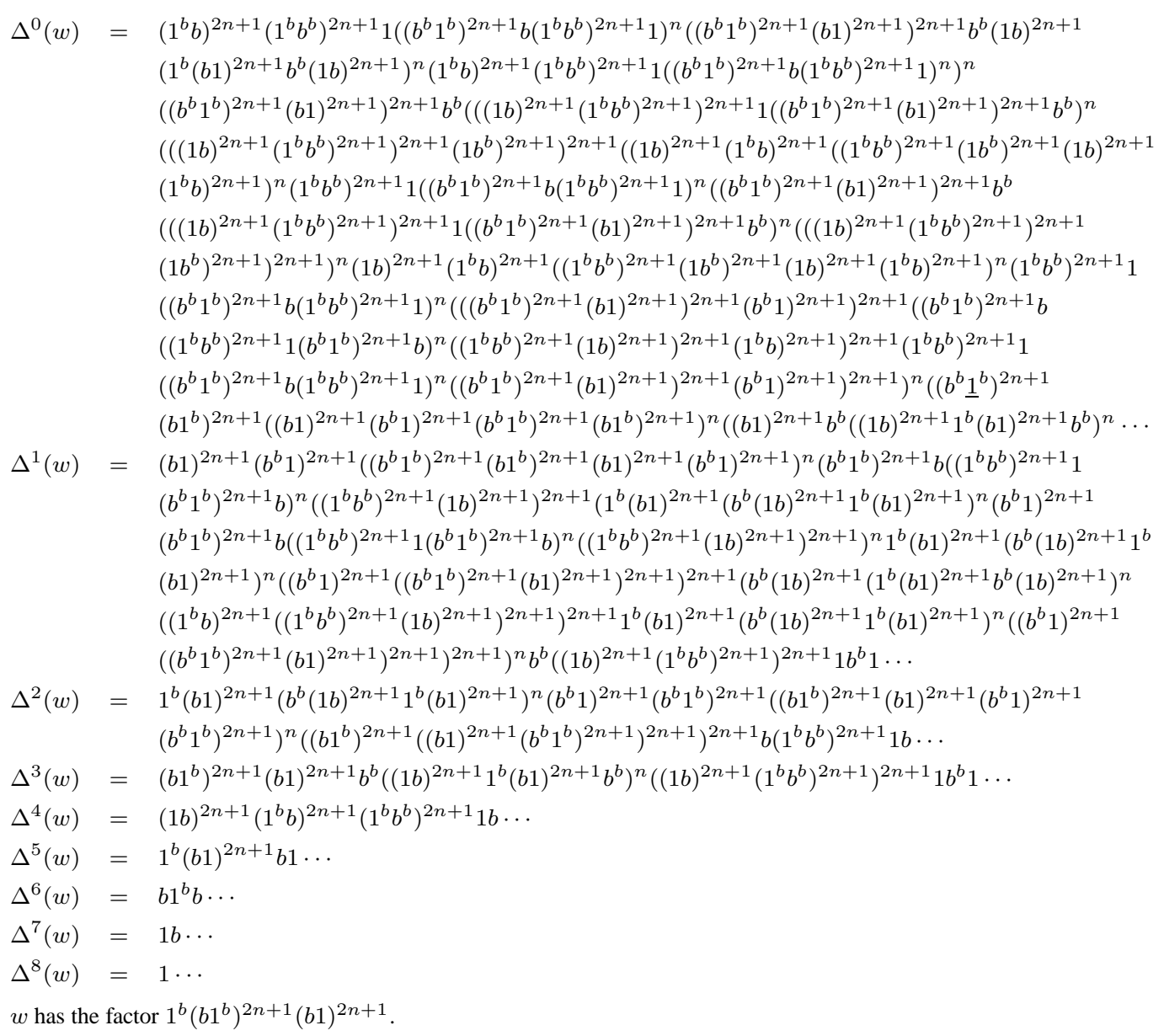

Case (20) If $p=1 b 1 b 11 b 1 b$, there are two subcases to consider.

(i) If $b=2$, then

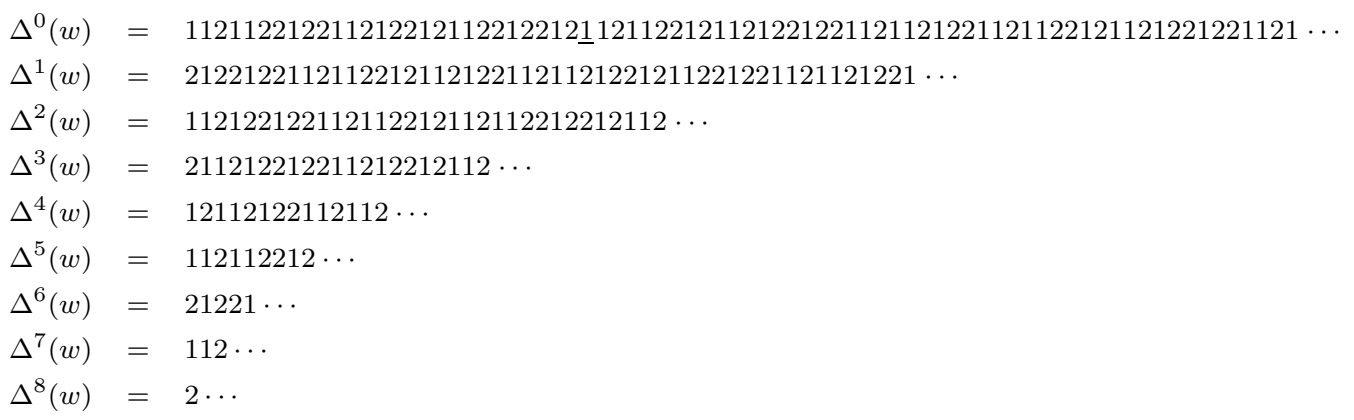

$w$ has the factor 1121122121 . 
(ii) If $b \neq 2$, then $n \neq 0$ and

$$
\begin{aligned}
& \Delta^{0}(w)=\left(1^{b} b\right)^{2 n+1}\left(1^{b} b^{b}\right)^{2 n+1} 1\left(\left(b^{b} 1^{b}\right)^{2 n+1} b\left(1^{b} b^{b}\right)^{2 n+1} 1\right)^{n}\left(( ( b ^ { b } 1 ^ { b } ) ^ { 2 n + 1 } ( b 1 ) ^ { 2 n + 1 } ) ^ { 2 n + 1 } b ^ { b } ( 1 b ) ^ { 2 n + 1 } \left(1^{b}\right.\right. \\
& \left.\left.(b 1)^{2 n+1} b^{b}(1 b)^{2 n+1}\right)^{n}\left(1^{b} b\right)^{2 n+1}\left(1^{b} b^{b}\right)^{2 n+1} 1\left(\left(b^{b} 1^{b}\right)^{2 n+1} b\left(1^{b} b^{b}\right)^{2 n+1} 1\right)^{n}\right)^{n} \\
& \left(\left(b^{b} 1^{b}\right)^{2 n+1}(b 1)^{2 n+1}\right)^{2 n+1} b^{b}\left(\left((1 b)^{2 n+1}\left(1^{b} b^{b}\right)^{2 n+1}\right)^{2 n+1} 1\left(\left(b^{b} 1^{b}\right)^{2 n+1}(b 1)^{2 n+1}\right)^{2 n+1} b^{b}\right)^{n} \\
& \left(\left((1 b)^{2 n+1}\left(1^{b} b^{b}\right)^{2 n+1}\right)^{2 n+1}\left(1 b^{b}\right)^{2 n+1}\right)^{2 n+1}(1 b)^{2 n+1}\left(\left(( \underline { 1 } ^ { b } b ) ^ { 2 n + 1 } \left(\left(1^{b} b^{b}\right)^{2 n+1}\right.\right.\right. \\
& \left.\left.(1 b)^{2 n+1}\right)^{2 n+1}\right)^{2 n+1} 1^{b} \ldots \\
& \Delta^{1}(w)=(b 1)^{2 n+1}\left(b^{b} 1\right)^{2 n+1}\left(\left(b^{b} 1^{b}\right)^{2 n+1}\left(b 1^{b}\right)^{2 n+1}(b 1)^{2 n+1}\left(b^{b} 1\right)^{2 n+1}\right)^{n}\left(b^{b} 1^{b}\right)^{2 n+1} b\left(\left(1^{b} b^{b}\right)^{2 n+1} 1\right. \\
& \left.\left(b^{b} 1^{b}\right)^{2 n+1} b\right)^{n}\left(( ( 1 ^ { b } b ^ { b } ) ^ { 2 n + 1 } ( 1 b ) ^ { 2 n + 1 } ) ^ { 2 n + 1 } 1 ^ { b } \left(( ( b 1 ) ^ { 2 n + 1 } ( b ^ { b } 1 ^ { b } ) ^ { 2 n + 1 } ) ^ { 2 n + 1 } b \left(\left(1^{b} b^{b}\right)^{2 n+1}\right.\right.\right. \\
& \left.\left.(1 b)^{2 n+1}\right)^{2 n+1} 1^{b}\right)^{n} \ldots \\
& \Delta^{2}(w)=1^{b}(b 1)^{2 n+1}\left(b^{b}(1 b)^{2 n+1} 1^{b}(b 1)^{2 n+1}\right)^{n}\left(b^{b} 1\right)^{2 n+1}\left(\left(b^{b} 1^{b}\right)^{2 n+1} b\left(\left(1^{b} b^{b}\right)^{2 n+1} 1\left(b^{b} 1^{b}\right)^{2 n+1} b\right)^{n}\right. \\
& \left.\left(\left(1^{b} b^{b}\right)^{2 n+1}(1 b)^{2 n+1}\right)^{2 n+1} 1^{b}(b 1)^{2 n+1}\left(b^{b}(1 b)^{2 n+1} 1^{b}(b 1)^{2 n+1}\right)^{n}\left(b^{b} 1\right)^{2 n+1}\right)^{n} \ldots \\
& \Delta^{3}(w)=\left(b 1^{b}\right)^{2 n+1}(b 1)^{2 n+1}\left(\left(b^{b} 1\right)^{2 n+1}\left(b^{b} 1^{b}\right)^{2 n+1}\left(b 1^{b}\right)^{2 n+1}(b 1)^{2 n+1}\right)^{n}\left(\left(b^{b} 1\right)^{2 n+1}\right. \\
& \left.\left(\left(b^{b} 1^{b}\right)^{2 n+1}(b 1)^{2 n+1}\right)^{2 n+1}\right)^{2 n+1} b^{b}(1 b)^{2 n+1} 1^{b} b \cdots \\
& \Delta^{4}(w)=(1 b)^{2 n+1} 1^{b}\left((b 1)^{2 n+1} b^{b}(1 b)^{2 n+1} 1^{b}\right)^{n}\left((b 1)^{2 n+1}\left(b^{b} 1^{b}\right)^{2 n+1}\right)^{2 n+1} b 1^{b} b \cdots \\
& \Delta^{5}(w)=\left(1^{b} b\right)^{2 n+1}\left(1^{b} b^{b}\right)^{2 n+1} 1 b \cdots \\
& \Delta^{6}(w)=(b 1)^{2 n+1} b^{b} 1 \cdots \\
& \Delta^{7}(w)=1^{b} b \cdots \\
& \Delta^{8}(w)=b \cdots
\end{aligned}
$$

Case (21) If $p=1 b 1 b 11 b b$, there are two subcases to consider.

(i) If $b=2$, there are again 3 subcases:

(a) If $p=1212112211$, then

$$
\begin{aligned}
\Delta^{0}(w)= & 11211221221121221211221221211212211211221221211221221121221211 \\
& 211221221211221221121221221121122121121221 \cdots \\
\Delta^{1}(w)= & 21221221121122121121122122121122122112112122121122122112122122122 \\
& 112112 \cdots \\
\Delta^{2}(w)= & 11212212211212212112212212112112212211212212 \cdots \\
\Delta^{3}(w)= & 21121221121122121121221221121 \cdots \\
\Delta^{4}(w)= & 1211221221121121221 \cdots \\
\Delta^{5}(w)= & 112212212112 \cdots \\
\Delta^{6}(w)= & 2212112 \cdots \\
\Delta^{7}(w)= & 2112 \cdots \\
\Delta^{8}(w)= & 12 \cdots \\
\Delta^{9}(w)= & 1 \cdots
\end{aligned}
$$

$w$ has the factor 11211221211. 
(b) If $p=1212112212$, then

$$
\begin{aligned}
& \Delta^{0}(w)= 112112212211212212112212212112122112112212212112212211212212112 \\
& 112212211212212211211212211211221221211211221211 \cdots \\
& \Delta^{1}(w)= 212212211211221211211221221211221221121121221 \\
& 2211212212112212212112122112112212212112 \cdots \\
& \Delta^{2}(w)= 11212212211212212112212212112122112112212112112212212112 \cdots \\
& \Delta^{3}(w)= 2112122112112212112112212211212212112 \cdots \\
& \Delta^{4}(w)= 121122122112122122112112 \cdots \\
& \Delta^{5}(w)= 112212211212212 \cdots \\
& \Delta^{6}(w)= 221221121 \cdots \\
& \Delta^{7}(w)= 21221 \cdots \\
& \Delta^{8}(w)= 112 \cdots \\
& \Delta^{9}(w)= 2 \cdots \\
&
\end{aligned}
$$

$w$ has the factor 1121121

(c) If $p=121211222$, then

$$
\begin{aligned}
\Delta^{0}(w)= & 112112212211212212112212212112122112112212212112212211212212211211 \\
& 21221211221221121 \cdots \\
\Delta^{1}(w)= & 212212211211221211211221221211221221121221211211221221 \cdots \\
\Delta^{2}(w)= & 11212212211212212112212211211212212 \cdots \\
\Delta^{3}(w)= & 21121221121122122121121 \cdots \\
\Delta^{4}(w)= & 121122122121121 \cdots \\
\Delta^{5}(w)= & 1122121121 \cdots \\
\Delta^{6}(w)= & 221121 \cdots \\
\Delta^{7}(w)= & 221 \cdots \\
\Delta^{8}(w)= & 2 \cdots
\end{aligned}
$$

$w$ has the factor 1121121 .

(ii) If $b \neq 2$, then $n \neq 0$ and

$$
\begin{aligned}
\Delta^{0}(w)= & \left(( 1 ^ { b } b ) ^ { 2 n + 1 } ( 1 ^ { b } b ^ { b } ) ^ { 2 n + 1 } 1 ( ( b ^ { b } 1 ^ { b } ) ^ { 2 n + 1 } b ( 1 ^ { b } b ^ { b } ) ^ { 2 n + 1 } 1 ) ^ { n } \left(\left(\left(b^{b} 1^{b}\right)^{2 n+1}(b 1)^{2 n+1}\right)^{2 n+1}\right.\right. \\
& \left.b^{b}(1 b)^{2 n+1}\left(1^{b}(b 1)^{2 n+1} b^{b}(1 b)^{2 n+1}\right)^{n}\left(1^{b} b\right)^{2 n+1}\left(1^{b} b^{b}\right)^{2 n+1} 1\left(\left(b^{b} 1^{b}\right)^{2 n+1} b\left(1^{b} b^{b}\right)^{2 n+1} 1\right)^{n}\right)^{n} \\
& \left(\left(b^{b} 1^{b}\right)^{2 n+1}(b 1)^{2 n+1}\right)^{2 n+1} b^{b}\left(\left((1 b)^{2 n+1}\left(1^{b} b^{b}\right)^{2 n+1}\right)^{2 n+1} 1\left(\left(b^{b} 1^{b}\right)^{2 n+1}(b 1)^{2 n+1}\right)^{2 n+1}\right. \\
& \left.b^{b}\right)^{n}\left(\left((1 b)^{2 n+1}\left(1^{b} b^{b}\right)^{2 n+1}\right)^{2 n+1}\left(1 b^{b}\right)^{2 n+1}\right)^{2 n+1}(1 b)^{2 n+1} 1^{b}\left((b 1)^{2 n+1} b^{b}(1 b)^{2 n+1} 1^{b}\right)^{n}\left(\left((b 1)^{2 n+1}\right.\right. \\
& \left.\left.\left(b^{b} 1^{b}\right)^{2 n+1}\right)^{2 n+1}\left(b 1^{b}\right)^{2 n+1}\right)^{2 n+1}(b 1)^{2 n+1} b^{b}\left((1 b)^{2 n+1} 1^{b}(b 1)^{2 n+1} b^{b}\right)^{n} \cdots \\
\Delta^{1}(w)= & \left(( b 1 ) ^ { 2 n + 1 } ( b ^ { b } 1 ) ^ { 2 n + 1 } ( ( b ^ { b } 1 ^ { b } ) ^ { 2 n + 1 } ( b 1 ^ { b } ) ^ { 2 n + 1 } ( b 1 ) ^ { 2 n + 1 } ( b ^ { b } 1 ) ^ { 2 n + 1 } ) ^ { n } ( b ^ { b } 1 ^ { b } ) ^ { 2 n + 1 } b \left(\left(1^{b} b^{b}\right)^{2 n+1}\right.\right. \\
& \left.\left.1\left(b^{b} 1^{b}\right)^{2 n+1} b\right)^{n}\left(\left(\left(1^{b} b^{b}\right)^{2 n+1}(1 b)^{2 n+1}\right)^{2 n+1}\left(1^{b} b\right)^{2 n+1}\right)^{2 n+1}\right)^{2 n+1} \ldots \\
\Delta^{2}(w)= & \left(1^{b}(b 1)^{2 n+1}\left(b^{b}(1 b)^{2 n+1} 1^{b}(b 1)^{2 n+1}\right)^{n}\left(\left(b^{b} 1\right)^{2 n+1}\left(\left(b^{b} 1^{b}\right)^{2 n+1}(b 1)^{2 n+1}\right)^{2 n+1}\right)^{2 n+1}\right)^{2 n+1} \ldots \\
\Delta^{3}(w)= & \left(\left(b 1^{b}\right)^{2 n+1}\left((b 1)^{2 n+1}\left(b^{b} 1^{b}\right)^{2 n+1}\right)^{2 n+1}\right)^{2 n+1} \ldots \\
\Delta^{4}(w)= & \left((1 b)^{2 n+1}\left(1^{b} b^{b}\right)^{2 n+1}\right)^{2 n+1} \ldots \\
\Delta^{5}(w)= & \left(1^{b} b^{b}\right)^{2 n+1} \ldots \\
\Delta^{6}(w)= & b^{b} \ldots \\
\Delta^{7}(w)= & b \cdots \\
w \text { has the factor } & 1^{b}\left(b 1^{b}\right)^{2 n+1}(b 1)^{2 n+1} .
\end{aligned}
$$


Case (22) If $p=1 b 1 b 1 b 11$, there are two subcases to consider.

(i) If $b=2$, then

$$
\begin{aligned}
\Delta^{0}(w) & =1121122122121122122112122122 \underline{1} 121122121121221 \cdots \\
\Delta^{1}(w) & =2122121122122112122122112112 \cdots \\
\Delta^{2}(w) & =112112212211212212 \cdots \\
\Delta^{3}(w) & =21221221121 \cdots \\
\Delta^{4}(w) & =1121221 \cdots \\
\Delta^{5}(w) & =2112 \cdots \\
\Delta^{6}(w) & =12 \cdots \\
\Delta^{7}(w) & =1 \cdots
\end{aligned}
$$

$w$ has the factor 1121122121

(ii) If $b \neq 2$, then $n \neq 0$ and

$$
\begin{aligned}
& \Delta^{0}(w)=\left(1^{b} b\right)^{2 n+1}\left(1^{b} b^{b}\right)^{2 n+1}\left(\left(1 b^{b}\right)^{2 n+1}(1 b)^{2 n+1}\left(1^{b} b\right)^{2 n+1}\left(1^{b} b^{b}\right)^{2 n+1}\right)^{n} 1\left(b^{b} 1^{b}\right)^{2 n+1}\left(b\left(1^{b} b^{b}\right)^{2 n+1}\right. \\
&\left.1\left(b^{b} \underline{1}^{b}\right)^{2 n+1}\right)^{n}\left(b 1^{b}\right)^{2 n+1}\left((b 1)^{2 n+1} b^{b}\left((1 b)^{2 n+1} 1^{b}(b 1)^{2 n+1} b^{b}\right)^{n}\left((1 b)^{2 n+1}\left(1^{b} b^{b}\right)^{2 n+1}\right)^{2 n+1}\right. \\
&\left.1\left(b^{b} 1^{b}\right)^{2 n+1}\left(b\left(1^{b} b^{b}\right)^{2 n+1} 1\left(b^{b} 1^{b}\right)^{2 n+1}\right)^{n}\left(b 1^{b}\right)^{2 n+1}\right)^{n} \cdots \\
& \Delta^{1}(w)=(b 1)^{2 n+1} b^{b}\left((1 b)^{2 n+1} 1^{b}(b 1)^{2 n+1} b^{b}\right)^{n}\left(1 b^{b}\right)^{2 n+1}(1 b)^{2 n+1}\left(\left(1^{b} b\right)^{2 n+1}\left(1^{b} b^{b}\right)^{2 n+1}\left(1 b^{b}\right)^{2 n+1}\right. \\
&\left.(1 b)^{2 n+1}\right)^{n}\left(\left(1^{b} b\right)^{2 n+1}\left(\left(1^{b} b^{b}\right)^{2 n+1}(1 b)^{2 n+1}\right)^{2 n+1}\right)^{2 n+1} 1^{b}(b 1)^{2 n+1} b^{b} 1 \cdots \\
& \Delta^{2}(w)=\left(1^{b} b\right)^{2 n+1}(1 b)^{2 n+1} 1^{b}\left((b 1)^{2 n+1} b^{b}(1 b)^{2 n+1} 1^{b}\right)^{n}\left((b 1)^{2 n+1}\left(b^{b} 1^{b}\right)^{2 n+1}\right)^{2 n+1} b 1^{b} b \cdots \\
& \Delta^{3}(w)=(b 1)^{2 n+1}\left(1^{b} b\right)^{2 n+1}\left(1^{b} b^{b}\right)^{2 n+1} 1 b \cdots \\
& \Delta^{4}(w)= 1^{b}(b 1)^{2 n+1} b^{b} 1 \cdots \\
& \Delta^{5}(w)= b 1^{b} b \cdots \\
& \Delta^{6}(w)= 1 b \cdots \\
& \Delta^{7}(w)= 1 \cdots \\
& w \text { has the factor } 1^{b}\left(b 1^{b}\right)^{2 n+1}(b 1)^{2 n+1 .} .
\end{aligned}
$$

Case (23) If $p=1 b 1 b 1 b 1 b$, there are two cases to consider.

(i) If $b=2$, then

$$
\begin{aligned}
\Delta^{0}(w) & =112112212212112212211212212112112212112122 \underline{1} 1211212212 \cdots \\
\Delta^{1}(w) & =21221211221221121121221121122121121 \cdots \\
\Delta^{2}(w) & =11211221221211221221121 \cdots \\
\Delta^{3}(w) & =21221211221221 \cdots \\
\Delta^{4}(w) & =112112212 \cdots \\
\Delta^{5}(w) & =21221 \cdots \\
\Delta^{6}(w) & =112 \cdots \\
\Delta^{7}(w) & =2 \cdots
\end{aligned}
$$

$w$ has the factor 1121121. 
(ii) If $b \neq 2$, then

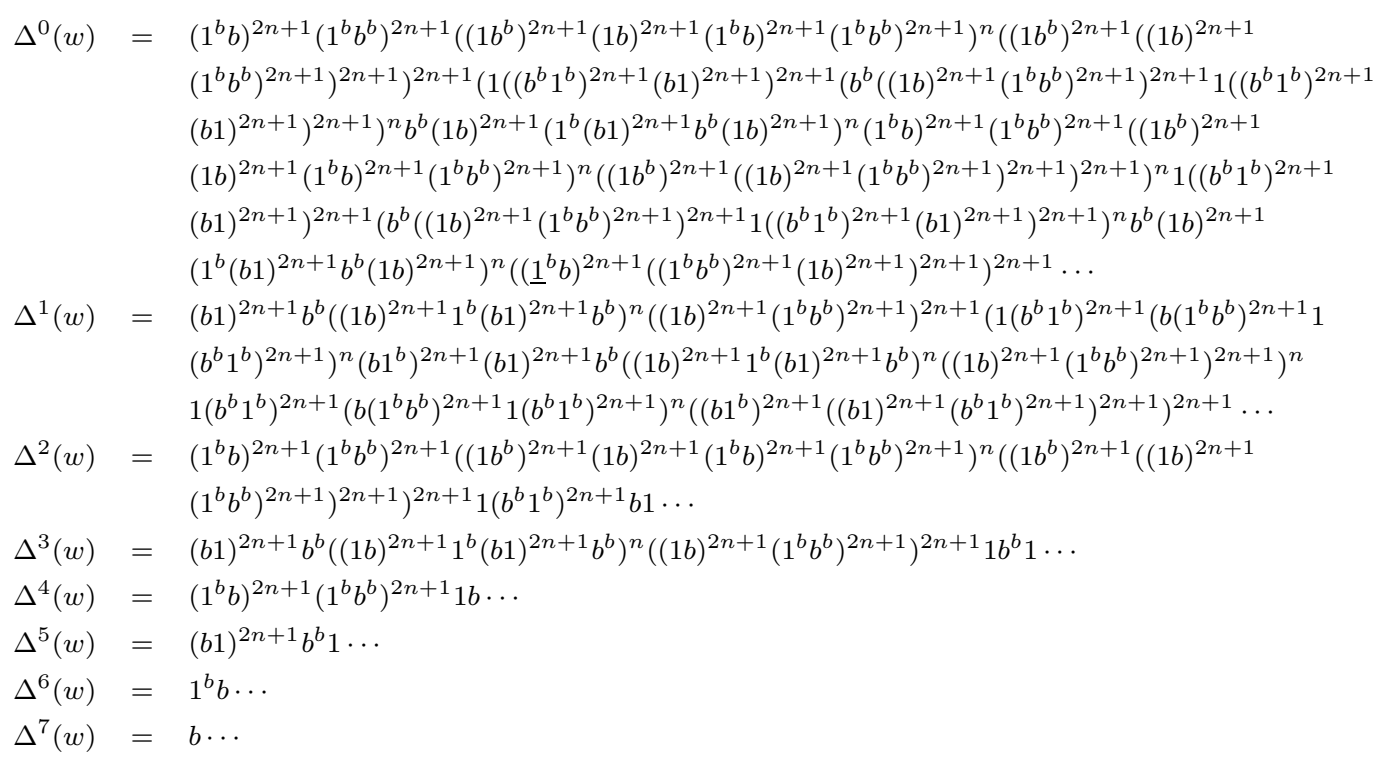

$w$ has the factor $\left(1^{b} b\right)^{2 n+1}\left(1^{b} b^{b}\right)^{2 n+1}(1 b)^{2 n+1}$.

Case (24) If $p=1 b 1 b 1 b b$, there are two subcases to consider.

(i) If $b=2$, then

$$
\begin{aligned}
\Delta^{0}(w) & =1121122122121122122 \underline{1} 121121221211221221 \cdots \\
\Delta^{1}(w) & =212212112212212112112212 \cdots \\
\Delta^{2}(w) & =1121122121121221 \cdots \\
\Delta^{3}(w) & =2122112112 \cdots \\
\Delta^{4}(w) & =112212 \cdots \\
\Delta^{5}(w) & =221 \cdots \\
\Delta^{6}(w) & =2 \cdots
\end{aligned}
$$

$w$ has the factor 1121121 .

(ii) If $b \neq 2$, then

$$
\begin{aligned}
& \Delta^{0}(w)=\left(( 1 ^ { b } b ) ^ { 2 n + 1 } ( 1 ^ { b } b ^ { b } ) ^ { 2 n + 1 } ( ( 1 b ^ { b } ) ^ { 2 n + 1 } ( 1 b ) ^ { 2 n + 1 } ( 1 ^ { b } b ) ^ { 2 n + 1 } ( 1 ^ { b } b ^ { b } ) ^ { 2 n + 1 } ) ^ { n } \left(( 1 b ^ { b } ) ^ { 2 n + 1 } \left((1 b)^{2 n+1}\right.\right.\right. \\
&\left.\left.\left(1^{b} b^{b}\right)^{2 n+1}\right)^{2 n+1}\right)^{2 n+1} 1\left(b^{b} 1^{b}\right)^{2 n+1}\left(b\left(1^{b} b^{b}\right)^{2 n+1} 1\left(b^{b} 1^{b}\right)^{2 n+1}\right)^{n}\left(( b 1 ^ { b } ) ^ { 2 n + 1 } \left((b 1)^{2 n+1} \ldots\right.\right. \\
& \Delta^{1}(w)=\left((b 1)^{2 n+1} b^{b}\left((1 b)^{2 n+1} 1^{b}(b 1)^{2 n+1} b^{b}\right)^{n}\left(\left((1 b)^{2 n+1}\left(1^{b} b^{b}\right)^{2 n+1}\right)^{2 n+1}\left(1 b^{b}\right)^{2 n+1}\right)^{2 n+1}\right)^{2 n+1} \ldots \\
& \Delta^{2}(w)=\left(\left(1^{b} b\right)^{2 n+1}\left(\left(1^{b} b^{b}\right)^{2 n+1}(1 b)^{2 n+1}\right)^{2 n+1}\right)^{2 n+1} \ldots \\
& \Delta^{3}(w)=\left((b 1)^{2 n+1}\left(b^{b} 1^{b}\right)^{2 n+1}\right)^{2 n+1} \ldots \\
& \Delta^{4}(w)=\left(1^{b} b^{b}\right)^{2 n+1} \ldots \\
& \Delta^{5}(w)= b^{b} \ldots \\
& \Delta^{6}(w)= b \cdots \\
& w \text { has the factor } 1^{b}\left(b 1^{b}\right)^{2 n+1}(b)^{2 n+1} .
\end{aligned}
$$

Case (25) If $p=1 b 1 b b 111$, there are two subcases to consider. 
(i) If $b=2$, there are again 2 subcases to consider.

(a) If $p=121221111$, then

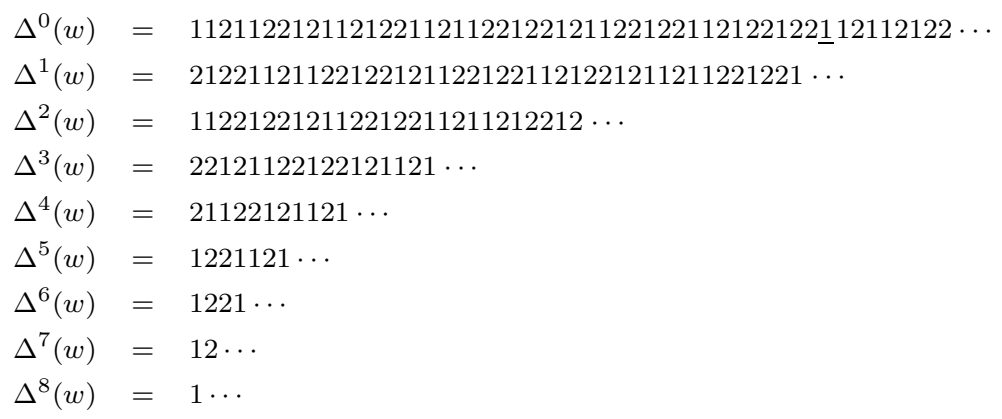

$w$ has the factor 1121121 .

(b) If $p=121221112$, then

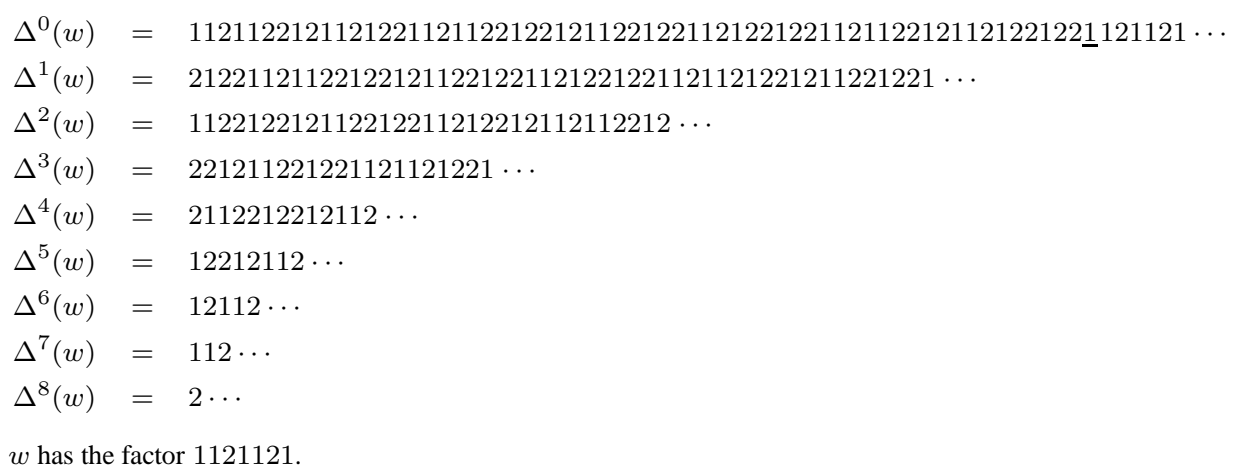

(ii) If $b \neq 2$, then

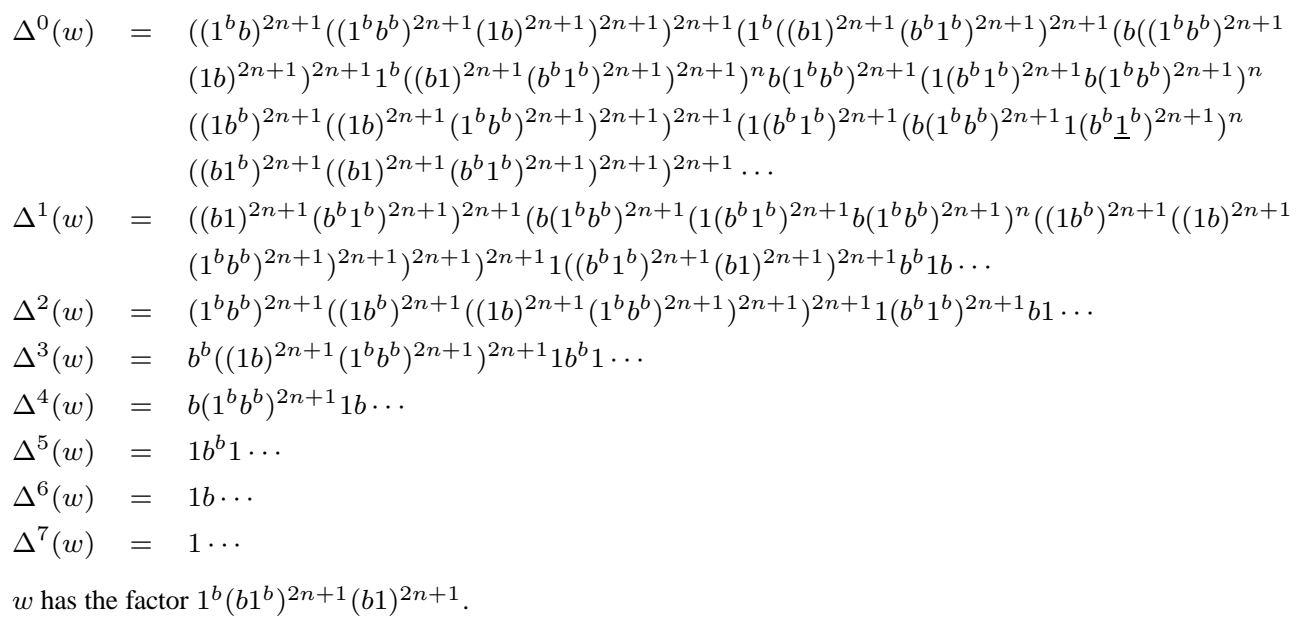

Case (26) If $p=1 b 1 b b 11 b$, there are two subcases to consider. 
(i) If $b=2$, then

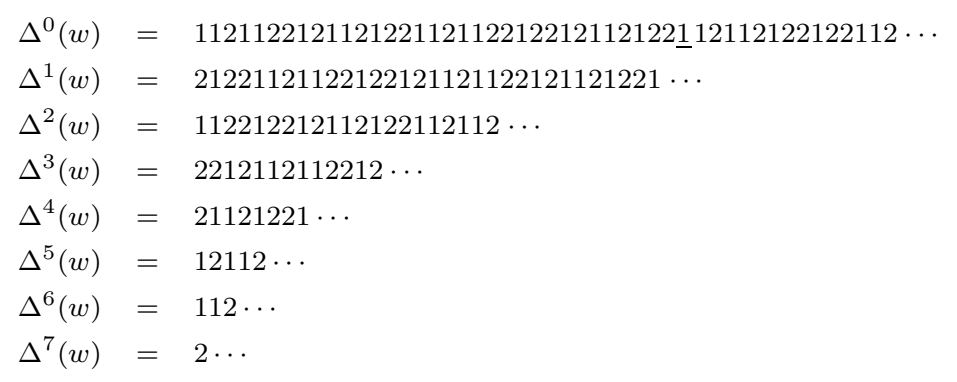

$w$ has the factor 1121121.

(ii) If $b \neq 2$, then

$$
\begin{aligned}
& \Delta^{0}(w)=\left(\left(1^{b} b\right)^{2 n+1}\left(\left(1^{b} b^{b}\right)^{2 n+1}(1 b)^{2 n+1}\right)^{2 n+1}\right)^{2 n+1} 1^{b}\left((b 1)^{2 n+1}\left(b^{b} 1^{b}\right)^{2 n+1}\right)^{2 n+1} \\
& \left(b\left(\left(1^{b} b^{b}\right)^{2 n+1}(1 b)^{2 n+1}\right)^{2 n+1} 1^{b}\left((b 1)^{2 n+1}\left(b^{b} 1^{b}\right)^{2 n+1}\right)^{2 n+1}\right)^{n}\left(b ( 1 ^ { b } b ^ { b } ) ^ { 2 n + 1 } \left(1\left(b^{b} 1^{b}\right)^{2 n+1} b\right.\right. \\
& \left.\left(1^{b} b^{b}\right)^{2 n+1}\right)^{n}\left(1 b^{b}\right)^{2 n+1}(1 b)^{2 n+1}\left(\left(1^{b} b\right)^{2 n+1}\left(1^{b} b^{b}\right)^{2 n+1}\left(1 b^{b}\right)^{2 n+1}(1 b)^{2 n+1}\right)^{n}\left(\left(1^{b} b\right)^{2 n+1}\right. \\
& \left.\left(\left(1^{b} b^{b}\right)^{2 n+1}(1 b)^{2 n+1}\right)^{2 n+1}\right)^{2 n+1} 1^{b}\left((b 1)^{2 n+1}\left(b^{b} 1^{b}\right)^{2 n+1}\right)^{2 n+1}\left(b\left(\left(1^{b} b^{b}\right)^{2 n+1}(1 b)^{2 n+1}\right)^{2 n+1} 1^{b}\right. \\
& \left.\left.\left((b 1)^{2 n+1}\left(b^{b} 1^{b}\right)^{2 n+1}\right)^{2 n+1}\right)^{n}\right)^{n} b\left(1^{b} b^{b}\right)^{2 n+1}\left(1\left(b^{b} 1^{b}\right)^{2 n+1} b\left(1^{b} b^{b}\right)^{2 n+1}\right)^{n}\left(1 b^{b}\right)^{2 n+1}\left((1 b)^{2 n+1} 1^{b}\right. \\
& \left((b 1)^{2 n+1} b^{b}(1 b)^{2 n+1} 1^{b}\right)^{n}\left((b 1)^{2 n+1}\left(b^{b} 1^{b}\right)^{2 n+1}\right)^{2 n+1} b\left(1^{b} b^{b}\right)^{2 n+1}\left(1\left(b^{b} 1^{b}\right)^{2 n+1}\right. \\
& \left.\left.b\left(1^{b} b^{b}\right)^{2 n+1}\right)^{n}\left(1 b^{b}\right)^{2 n+1}\right)^{n}(1 b)^{2 n+1} 1^{b}\left((b 1)^{2 n+1} b^{b}(1 b)^{2 n+1} 1^{b}\right)^{n}\left(\left((b 1)^{2 n+1}\left(b^{b} \underline{1}^{b}\right)^{2 n+1}\right)^{2 n+1}\right. \\
& \left.\left(b 1^{b}\right)^{2 n+1}\right)^{2 n+1}(b 1)^{2 n+1} b^{b}\left((1 b)^{2 n+1} 1^{b}(b 1)^{2 n+1} b^{b}\right)^{n}\left(\left((1 b)^{2 n+1}\left(1^{b} b^{b}\right)^{2 n+1}\right)^{2 n+1}\left(1 b^{b}\right)^{2 n+1}\right)^{2 n+1} \ldots \\
& \Delta^{1}(w)=\left((b 1)^{2 n+1}\left(b^{b} 1^{b}\right)^{2 n+1}\right)^{2 n+1} b\left(1^{b} b^{b}\right)^{2 n+1}\left(1\left(b^{b} 1^{b}\right)^{2 n+1} b\left(1^{b} b^{b}\right)^{2 n+1}\right)^{n}\left(\left(1 b^{b}\right)^{2 n+1}(1 b)^{2 n+1} 1^{b}\right. \\
& \left.\left((b 1)^{2 n+1} b^{b}(1 b)^{2 n+1} 1^{b}\right)^{n}\left((b 1)^{2 n+1}\left(b^{b} 1^{b}\right)^{2 n+1}\right)^{2 n+1} b\left(1^{b} b^{b}\right)^{2 n+1}\left(1\left(b^{b} 1^{b}\right)^{2 n+1} b\left(1^{b} b^{b}\right)^{2 n+1}\right)^{n}\right)^{n} \\
& \left(1 b^{b}\right)^{2 n+1}(1 b)^{2 n+1}\left(( 1 ^ { b } b ) ^ { 2 n + 1 } ( 1 ^ { b } b ^ { b } ) ^ { 2 n + 1 } ( 1 b ^ { b } ) ^ { 2 n + 1 } ( 1 b ) ^ { 2 n + 1 } \left(( 1 ^ { b } b ) ^ { 2 n + 1 } \left(\left(1^{b} b^{b}\right)^{2 n+1}\right.\right.\right. \\
& \left.\left.(1 b)^{2 n+1}\right)^{2 n+1}\right)^{2 n+1} 1^{b}(b 1)^{2 n+1} b^{b} 1 \\
& \Delta^{2}(w)=\left(1^{b} b^{b}\right)^{2 n+1}\left(1 b^{b}\right)^{2 n+1}\left((1 b)^{2 n+1}\left(1^{b} b\right)^{2 n+1}\left(1^{b} b^{b}\right)^{2 n+1}\left(1 b^{b}\right)^{2 n+1}\right)^{n}(1 b)^{2 n+1} 1^{b}\left((b 1)^{2 n+1}\right. \\
& \left.b^{b}(1 b)^{2 n+1} 1^{b}\right)^{n}\left((b 1)^{2 n+1}\left(b^{b} 1^{b}\right)^{2 n+1}\right)^{2 n+1} b 1^{b} b \cdots \\
& \Delta^{3}(w)=b^{b}(1 b)^{2 n+1}\left(1^{b}(b 1)^{2 n+1} b^{b}(1 b)^{2 n+1}\right)^{n}\left(1^{b} b\right)^{2 n+1}\left(1^{b} b^{b}\right)^{2 n+1} 1 b \cdots \\
& \Delta^{4}(w)=\left(b 1^{b}\right)^{2 n+1}(b 1)^{2 n+1} b^{b} 1 \cdots \\
& \Delta^{5}(w)=(1 b)^{2 n+1} 1^{b} b \cdots \\
& \Delta^{6}(w)=1^{b} b \cdots \\
& \Delta^{7}(w)=b \cdots
\end{aligned}
$$

Case (27) If $p=1 b 1 b b b$, there are two subcases to consider. 
(i) If $b \neq 2$, then

$$
\begin{aligned}
\Delta^{0}(w)= & \left(\left(1^{b} b\right)^{2 n+1}\left(\left(1^{b} b^{b}\right)^{2 n+1}(1 b)^{2 n+1}\right)^{2 n+1}\right)^{2 n+1} 1^{b}(b 1)^{2 n+1}\left(b^{b}(1 b)^{2 n+1} 1^{b}(b 1)^{2 n+1}\right)^{n} \\
& \left(\left(\left(b^{b} 1\right)^{2 n+1}\left(\left(b^{b} 1^{b}\right)^{2 n+1}(b 1)^{2 n+1}\right)^{2 n+1}\right)^{2 n+1} b^{b}(1 b)^{2 n+1}\left(1^{b}(b 1)^{2 n+1} b^{b}(1 b)^{2 n+1}\right)^{n}\right. \\
& \left.\left(\left(\underline{1}^{b} b\right)^{2 n+1}\left(\left(1^{b} b^{b}\right)^{2 n+1}(1 b)^{2 n+1}\right)^{2 n+1}\right)^{2 n+1} 1^{b}(b 1)^{2 n+1}\left(b^{b}(1 b)^{2 n+1} 1^{b}(b 1)^{2 n+1}\right)^{n}\right)^{n} \\
& \left(b^{b} 1\right)^{2 n+1}\left(b^{b} 1^{b}\right)^{2 n+1} b 1^{b} b \cdots \\
\Delta^{1}(w)= & \left(\left((b 1)^{2 n+1}\left(b^{b} 1^{b}\right)^{2 n+1}\right)^{2 n+1}\left(b 1^{b}\right)^{2 n+1}\right)^{2 n+1}(b 1)^{2 n+1} b^{b} 1 b \cdots \\
\Delta^{2}(w)= & \left(\left(1^{b} b^{b}\right)^{2 n+1}(1 b)^{2 n+1}\right)^{2 n+1} 1^{b} b 1 \cdots \\
\Delta^{3}(w)= & \left(b^{b} 1^{b}\right)^{2 n+1} b 1 \cdots \\
\Delta^{4}(w)= & b^{b} 1 \cdots \\
\Delta^{5}(w)= & b \cdots
\end{aligned}
$$

$w$ has the factor

$$
\begin{aligned}
\left(\left(1^{b} b\right)^{2 n+1}\left(\left(1^{b} b^{b}\right)^{2 n+1}(1 b)^{2 n+1}\right)^{2 n+1}\right)^{2 n+1} & 1^{b}(b 1)^{2 n+1} \\
& \left.\left(b^{b}(1 b)^{2 n+1} 1^{b}(b 1)^{2 n+1}\right)^{n}\right)^{n}\left(b^{b} 1\right)^{2 n+1}\left(b^{b} 1^{b}\right)^{2 n+1} b 1^{b} .
\end{aligned}
$$

(ii) If $b=2$, there are again 3 subcases to consider.

(a) If $p=12122211$, then

$$
\begin{aligned}
& \Delta^{0}(w)=112112212112122122 \underline{1} 1211221211212211 \cdots \\
& \Delta^{1}(w)=21221121121221221121122 \ldots \\
& \Delta^{2}(w)=112212112122122 \ldots \\
& \Delta^{3}(w)=2211211212 \cdots \\
& \Delta^{4}(w)=221211 \cdots \\
& \Delta^{5}(w)=2112 \cdots \\
& \Delta^{6}(w)=12 \cdots \\
& \Delta^{7}(w)=1 \cdots
\end{aligned}
$$

$w$ has the factor 11211221211212211.

(b) If $p=12122212$, then

$$
\begin{aligned}
& \Delta^{0}(w)=1121122121121221221121122121121122122 \cdots \\
& \Delta^{1}(w)=212211211212212211212212 \cdots \\
& \Delta^{2}(w)=11221211212211211 \cdots \\
& \Delta^{3}(w)=22112112212 \cdots \\
& \Delta^{4}(w)=2212211 \cdots \\
& \Delta^{5}(w)=2122 \cdots \\
& \Delta^{6}(w)=112 \cdots \\
& \Delta^{7}(w)=2 \cdots
\end{aligned}
$$

$w$ has the factor 11211221211211. 
(c) If $p=1212222$, then

$$
\begin{aligned}
& \Delta^{0}(w)=112112212112122122 \underline{1} 1211212211 \cdots \\
& \Delta^{1}(w)=2122112112122121122 \cdots \\
& \Delta^{2}(w)=1122121121122 \cdots \\
& \Delta^{3}(w)=22112122 \cdots \\
& \Delta^{4}(w)=22112 \cdots \\
& \Delta^{5}(w)=221 \cdots \\
& \Delta^{6}(w)=2 \cdots
\end{aligned}
$$

$w$ has the factor 1121121 .

Case (28) If $p=1 b b 11 b$, then

$$
\begin{aligned}
\Delta^{0}(w)= & \left(1^{b} b^{b}\right)^{2 n+1}\left(1 b^{b}\right)^{2 n+1}\left((1 b)^{2 n+1}\left(1^{b} b\right)^{2 n+1}\left(1^{b} b^{b}\right)^{2 n+1}\left(1 b^{b}\right)^{2 n+1}\right)^{n}(1 b)^{2 n+1} 1^{b} \\
& \left((b 1)^{2 n+1} b^{b}(1 b)^{2 n+1} 1^{b}\right)^{n}\left((b 1)^{2 n+1}\left(b^{b} 1^{b}\right)^{2 n+1}\right)^{2 n+1} b 1^{b} b \cdots \\
\Delta^{1}(w)= & b^{b}(1 b)^{2 n+1}\left(1^{b}(b 1)^{2 n+1} b^{b}(1 b)^{2 n+1}\right)^{n}\left(1^{b} b\right)^{2 n+1}\left(1^{b} b^{b}\right)^{2 n+1} 1 b \cdots \\
\Delta^{2}(w)= & \left(b 1^{b}\right)^{2 n+1}(b 1)^{2 n+1} b^{b} 1 \cdots \\
\Delta^{3}(w)= & (1 b)^{2 n+1} 1^{b} b \cdots \\
\Delta^{4}(w)= & 1^{b} b \cdots \\
\Delta^{5}(w)= & b \cdots
\end{aligned}
$$

$w$ has the factor $1^{b} b 1^{b}$.

Case (29) If $p=1 b b 1 b$, then

$$
\begin{aligned}
\Delta^{0}(w) & =\left(1^{b} b^{b}\right)^{2 n+1} 1\left(\left(b^{b} 1^{b}\right)^{2 n+1} b\left(1^{b} b^{b}\right)^{2 n+1} 1\right)^{n}\left(\left(b^{b} \underline{1}^{b}\right)^{2 n+1}(b 1)^{2 n+1}\right)^{2 n+1} b^{b} 1 b \cdots \\
\Delta^{1}(w) & =\left(b^{b} 1\right)^{2 n+1}\left(b^{b} 1^{b}\right)^{2 n+1} b 1 \cdots \\
\Delta^{2}(w) & =(b 1)^{2 n+1} b^{b} 1 \cdots \\
\Delta^{3}(w) & =1^{b} b \cdots \\
\Delta^{4}(w) & =b \cdots
\end{aligned}
$$

$w$ has the factor $1^{b} b 1$. 